ARTICLE

\title{
Leveraging machine learning essentiality predictions and chemogenomic interactions to identify antifungal targets
}

Ci Fu ${ }^{1,9}$, Xiang Zhang (2) ${ }^{2}$, Amanda O. Veri1,9, Kali R. Iyer ${ }^{1,9}$, Emma Lash ${ }^{1,9}$, Alice Xue ${ }^{1,9}$, Huijuan Yan ${ }^{3}$, Nicole M. Revie ${ }^{1}$, Cassandra Wong ${ }^{4}$, Zhen-Yuan Lin ${ }^{4}$, Elizabeth J. Polvi ${ }^{1}$, Sean D. Liston (10 1, Benjamin VanderSluis (10 2, Jing Hou ${ }^{1,5}$, Yoko Yashiroda ${ }^{6}$, Anne-Claude Gingras (1) 1,4, Charles Boone (i) 1,5,6, Teresa R. O'Meara (i) ${ }^{7}$, Matthew J. O'Meara (i) ${ }^{8}$, Suzanne Noble ${ }^{3}$, Nicole Robbins ${ }^{1}$, Chad L. Myers (i) ${ }^{2 凶} \&$ Leah E. Cowen (iD ${ }^{1 凶}$

Fungal pathogens pose a global threat to human health, with Candida albicans among the leading killers. Systematic analysis of essential genes provides a powerful strategy to discover potential antifungal targets. Here, we build a machine learning model to generate genomewide gene essentiality predictions for $\mathrm{C}$. albicans and expand the largest functional genomics resource in this pathogen (the GRACE collection) by 866 genes. Using this model and chemogenomic analyses, we define the function of three uncharacterized essential genes with roles in kinetochore function, mitochondrial integrity, and translation, and identify the glutaminyl-tRNA synthetase $\mathrm{G} \ln 4$ as the target of $\mathrm{N}$-pyrimidinyl- $\beta$-thiophenylacrylamide (NP-BTA), an antifungal compound.

\footnotetext{
${ }^{1}$ Department of Molecular Genetics, University of Toronto, Toronto, ON M5G 1M1, Canada. ${ }^{2}$ Department of Computer Science and Engineering, University of Minnesota, Minneapolis, MN 55455, USA. ${ }^{3}$ Department of Microbiology and Immunology, UCSF School of Medicine, San Francisco, CA 94143, USA.

${ }^{4}$ Lunenfeld-Tanenbaum Research Institute, Sinai Health System, Toronto, ONM5G 1X5Canada. ${ }^{5}$ Donnelly Centre, University of Toronto, Toronto, ON M5S 3E1, Canada. ${ }^{6}$ RIKEN Center for Sustainable Resource Science, Wako, Saitama 351-0198, Japan. ${ }^{7}$ Department of Microbiology and Immunology, University of Michigan Medical School, Ann Arbor, MI 48109, USA. ${ }^{8}$ Department of Computational Medicine and Bioinformatics, University of Michigan, Ann Arbor, MI 48109, USA. ${ }^{9}$ These authors contributed equally: Ci Fu, Xiang Zhang, Amanda O. Veri, Kali R. Iyer, Emma Lash, Alice Xue. ${ }^{凶}$ email: chadm@umn.edu; leah.cowen@utoronto.ca
} 
W hile modern medicine has enabled survival of maladies that would otherwise have been lethal, this has been accompanied by an expansion of immunosuppressed patient populations vulnerable to pathogenic microbes. Among the microbial threats, fungi have emerged as a leading cause of human disease, particularly in immunocompromised individuals ${ }^{1-3}$. These opportunistic invaders include Candida species, which are the primary cause of systemic fungal infection in North America, with devastating mortality rates of $\sim 40 \%{ }^{3,4}$. While Candida albicans is the leading causal agent of these infections, there has been an alarming increase in drug-resistant non-albicans species ${ }^{5-7}$. Treatment of these infections is hindered by the limited antifungal armamentarium with only three main drug classes available for clinical use: azoles, polyenes, and echinocandins. Both azoles and polyenes target the membrane sterol ergosterol, with azoles inhibiting its biosynthesis and polyenes extracting the essential sterol from cell membranes ${ }^{8,9}$. Echinocandins inhibit synthesis of $\beta$ - $(1,3)$ glucan, a key component of fungal cell walls ${ }^{8,9}$. Thus, there is a pressing need to identify additional targets to bolster the antifungal pipeline.

The vast majority of antimicrobial agents in clinical use target functions essential for pathogen viability. This includes azoles and echinocandins, which target the essential gene products Erg11 and Fks1, respectively ${ }^{8,9}$. Essential genes that are fungal-specific or have limited conservation in humans provide attractive targets for selectively killing the pathogen ${ }^{10}$, motivating systematic analysis of genes required for fungal survival to expand the target space for drug development. Functional genomic analyses in the model yeast Saccharomyces cerevisiae have established extensive functional redundancy and buffering, as only $\sim 17 \%$ of genes in $S$. cerevisiae are essential under standard laboratory conditions ${ }^{11,12}$. Extrapolation to fungal pathogens is limited as $\sim 40 \%$ of C. albicans genes lack identifiable homologs in S. cerevisiae and $~ 19 \%$ of C. albicans predicted proteins display no significant similarity to proteins from other organisms ${ }^{13}$.

Functional genomic analysis in C. albicans provides unprecedented power for identifying additional therapeutic targets. Traditionally, large-scale genetic studies were hampered by the organism's diploid genome and lack of a sexual cycle ${ }^{14-16}$. One of the foundational systematic screens focused on identifying genes required for pathogenicity in a mouse and exploited a library of homozygous deletion mutants covering $\sim 11 \%$ of the genome ${ }^{17}$. More recently, a stable haploid C. albicans isolate was employed to generate a genome-wide transposon mutant collection that was evaluated for gene essentiality ${ }^{18}$. Finally, a functional genomics resource termed the gene replacement and conditional expression (GRACE) collection was generated to define gene function in $C$. albicans ${ }^{19}$. In this mutant library, each strain carries a precise replacement of one of the two alleles of a gene with an auxotrophic marker, while the remaining allele is under the control of a tetracycline-repressible promoter such that gene expression can be repressed by the tetracycline analog doxycycline $(\mathrm{DOX})^{19}$. Prior to this study, the GRACE collection included 2357 strains covering 2327 genes and was enriched for orthologs of essential genes in S. cerevisiae or the fission yeast Schizosaccharomyces pombe ${ }^{19}$. In vitro screening of the GRACE collection identified 634 strains with a severe or complete growth defect following target gene repression, and established that the predictive value of S. cerevisiae essentiality was limited to $52.4 \%{ }^{20}$, emphasizing the necessity of defining the essential gene set directly in the pathogen.

Here, we established a machine-learning model to provide a comprehensive essentiality prediction for the $\sim 6500$ genes annotated in the $C$. albicans genome ${ }^{21}$. Predictions from this model guided the generation of 866 additional GRACE mutants (termed GRACEv2), including 115 strains for predicted essential genes, further expanding this functional genomics resource to provide $\sim 48 \%$ coverage of the $C$. albicans genome. By coupling our computational prediction with this expanded GRACE collection, we identified 149 fungal-specific essential genes. Integrating our machine-learning model with phylogenetic analysis and coexpression datasets, we described the function of several previously uncharacterized genes involved in kinetochore function $\left(C 1 \_01070 \mathrm{C}\right.$ or KRP1), mitochondrial integrity (C6_03200W or EMF1), and translation initiation (C2_04370W or TIF33). Finally, we identified $\mathrm{N}$-pyrimidinyl-beta-thiophenylacrylamide (NP-BTA) as a molecule with potent activity against $C$. albicans that inhibits the essential glutaminyl-tRNA synthetase Gln4. Collectively, this work leverages machine learning, functional genomics, and chemical genomics to characterize essential genes and defines additional targets to advance antifungal drug development.

\section{Results}

Leveraging a machine-learning model to generate genome-wide predictions of $\boldsymbol{C}$. albicans gene essentiality. In order to generate a comprehensive assessment of genes with evidence for essentiality in C. albicans, we first collected a set of functional genomic features for use in training a machine-learning model (Supplementary Data 1). These included features derived from a collection of gene expression datasets such as gene expression level (Transcripts Per Kilobase Million (TPM) median), gene expression variance, and degree of co-expression (number of partners in a co-expression network $)^{22}$. It also encompassed a codon adaptation index (CAI), which measures the bias in codon usage across each gene ${ }^{21}$; the number of SNPs per nucleotide for each gene across a set of sequenced $C$. albicans strains ${ }^{23}$; the presence of an essential ortholog in S. cerevisiae ${ }^{12}$; and the presence of a duplicated set of paralogs in S. cerevisiae that exhibited a synthetic sick/lethal genetic interaction ${ }^{24}$. Finally, we incorporated six features from a recent transposon mutagenesis (TnSeq) study in a stable haploid background for which a previous machine-learning model was developed ${ }^{18}$ (Fig. 1a).

With this collection of features, we built a supervised machinelearning model based on random forests (RF) ${ }^{25}$ using the $C$. albicans GRACE mutant library as a gold standard for gene essentiality ${ }^{19}$. We confirmed the essentiality of all GRACE strains by pre-treating mutants with a high concentration $(100 \mu \mathrm{g} / \mathrm{mL})$ of DOX before transfer onto solid medium containing $100 \mu \mathrm{g} / \mathrm{mL}$ DOX and allowing cells to grow for $48 \mathrm{~h}$. Colony images were qualitatively scored for growth defects using a scale from 0 (growth comparable to wild type) to 4 (no growth) by two independent researchers (Supplementary Fig. 1a and Supplementary Data 2). A gene was classified as essential if its corresponding GRACE strain was able to grow robustly in the absence of DOX but displayed a severe growth defect in the presence of DOX. In instances where discrepant results were obtained between researchers, essentiality was further characterized by spot dilution assays (Supplementary Fig. 1b). This rigorous assessment ensured that an essential designation was restricted to genes for which depletion resulted in very little to no growth (score of 3 or 4), ensuring that genes for which transcriptional repression merely resulted in modest growth defects (score of 1 or 2) were not falsely identified. Scores were also assigned to strains with growth defects that did not meet essentiality criteria (Supplementary Data 2). While this strict approach may have resulted in the omission of genes previously characterized as essential ${ }^{26,27}$, this assessment of essentiality provided a robust training set for our machine-learning model, which included 523 confirmed essential genes and 1804 non-essential genes. We trained our RF classifier on $80 \%$ of this GRACE training set with fivefold cross-validation 
a

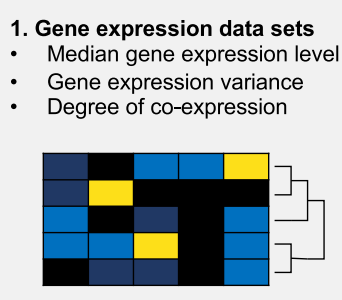

Essentiality training

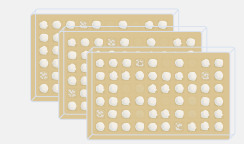

2,327 GRACE mutants: 523 essential

1,804 non-essential

\section{Input features}

2. Sequence features

- Codon adaptation index

- Sequence variation

AUG-GGA-GAC-CUG-ACC

Met-Gly-Asp-Lys-Thr

Met-Gly-Asp-Ser-Thr
3. S. cerevisiae data

- Ortholog essentiality

- Synthetic lethal/sick paralogs

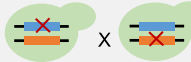

Viable

Viable

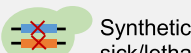
sick/lethal
4. Transposon essentiality dataset

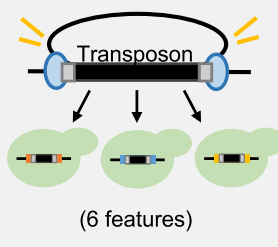

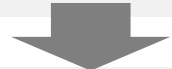

Random forest model

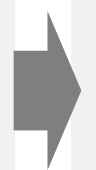<smiles>OOOOOO</smiles>

Tree 1

Predicted essentiality scores: 6,638 genes

\section{Experimental validation}
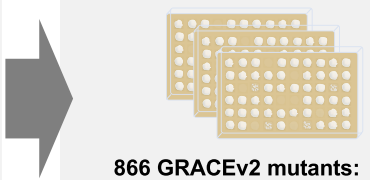

866 GRACEv2 mutants:

98 essential

768 non-essential b
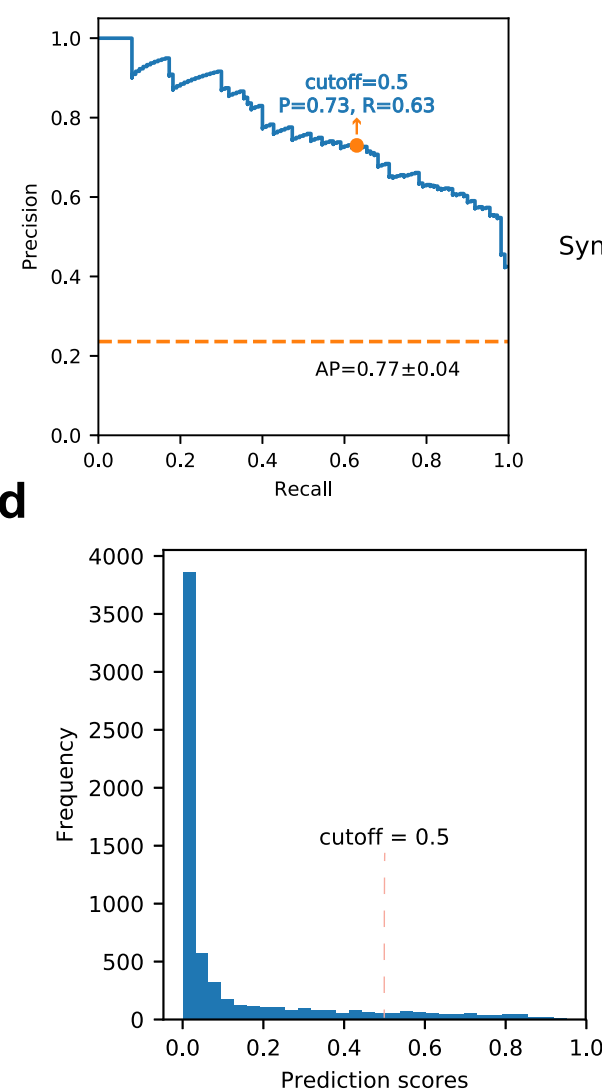

C

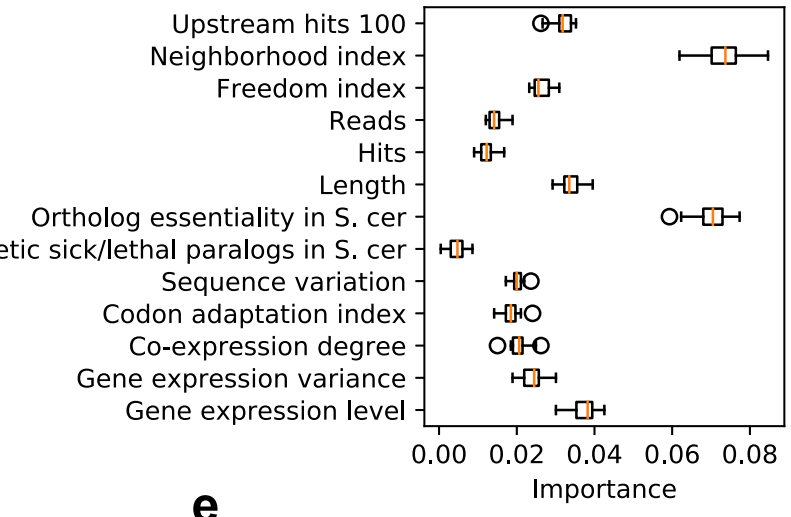

e

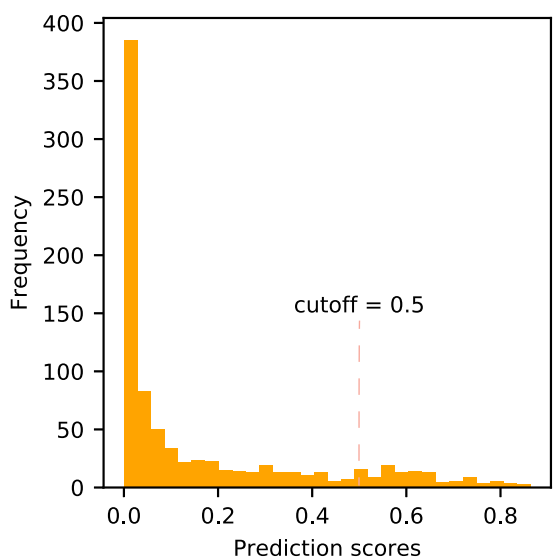

Fig. 1 Building a machine-learning model to predict essentiality and testing on the original GRACE collection. a Overview of the input, output, and validation process of our random forest model. b Precision-recall curve of our random forest model on $20 \%$ of the GRACE gene set. The model was trained and optimized on the other $80 \%$ of the GRACE gene set. The default stringent cutoff score for essential gene predictions results in a precision of 0.73 and a recall of 0.63 , with an average precision score of 0.77 . The error bars reflect the standard deviation across estimates derived from 10,000 different resamplings (with replacement) of the test set. c Permutation feature importance of our random forest model for the whole GRACE gene set. The decrease in a model upon permutation of that feature score reflects importance, and the box plots show variation for each feature's importance across 30 permutations. The whiskers extend out to 1.5 times the inter-quartile range, and the flier points reflect outliers beyond 1.5 times the inter-quartile range. S. cer represents S. cerevisiae. d Distribution of our random forest prediction scores across 6638 C. albicans genes. e Distribution of prediction scores for the 866 selected candidates for further experimental validation. Source data are provided as a Source Data file. 
for hyperparameter tuning, and then tested the performance on the remaining $20 \%$. We achieved an average precision (AP) of 0.77 and an area under the receiver operating characteristic curve (AUC) of 0.92 (Fig. $1 \mathrm{~b}$ and Supplementary Fig. 2b). Using a standard permutation-based approach ${ }^{25}$, we evaluated the relative importance of features contributing to the model's performance (Fig. 1c). This highlighted ortholog essentiality in S. cerevisiae and neighborhood index measure from the TnSeq screen ${ }^{18}$ as two of the most important features, although all included features contributed unique information (Fig. 1c, Supplementary Note 1 and Supplementary Table 1).

One defining strength of our machine-learning model is that it predicts essentiality for the vast majority of genes annotated in the C. albicans genome (6638 gene annotations were included in the model output; see "Methods" for details), making this the most comprehensive essentiality prediction dataset to date. Specifically, it includes predictions for 745 genes that were not addressed in other studies ${ }^{18}$. Using a stringent cutoff of essentiality (RF output score $>0.5$ ), our model predicted 654 genes in C. albicans to be essential (Fig. 1d and Supplementary Data 1). These were enriched in diverse processes including ribosome biogenesis, cellular metabolic processes, translation, RNA processing, transcription, mitotic cell cycle, and microtubule cytoskeleton organization $(\mathrm{FDR}<1 \%$; Supplementary Data 3).

Experimental validation of $C$. albicans essentiality predictions. To assess the accuracy of our prediction model, we selected 866 genes for validation, covering the range of RF prediction scores (Figs. 1e and 2a), including 115 genes with RF scores $>0.5$. For each selected gene, we constructed a corresponding GRACE strain, expanding the genome coverage of this functional genomics resource from 2327 to 3193 mutants or $48 \%$ of the $C$. albicans genome (GRACEv2 collection). We experimentally tested the essentiality of the GRACEv2 collection and for genes with an RF prediction score of $>0.5$, we confirmed essentiality of the corresponding mutants at a rate of $\sim 64 \%$ (74 out of 115 ) relative to a genome-wide expectation of $\sim 20 \% 12,18,20$. Of the remaining 41 genes predicted but not confirmed to be essential, 23 showed a slow growth phenotype (growth score of 1 or 2). Among the 866 strains tested in the GRACEv2 collection, 98 genes were confirmed as essential (Supplementary Data 1). We found that the RF prediction score was highly effective in identifying essential genes, resulting in an AP of 0.66 and AUC of 0.95 on the GRACEv2 validation set (Fig. $2 \mathrm{~b}$ and Supplementary Fig. 2c).

These assessments confirmed that use of a machine-learning model that robustly integrates numerous data sources substantially improves the accuracy of essential gene prediction over models based on individual features. For example, essentiality of orthologs in S. cerevisiae predicts essentiality in C. albicans with only $36 \%$ accuracy (or $46.7 \%$ accuracy if one includes C. albicans mutants with slow growth phenotypes), and synthetic lethality of duplicated paralogs in S. cerevisiae predicts essentiality with $31 \%$ accuracy (Supplementary Data 1). Also, these analyses allowed us to assess essentiality phenotypes of 43 genes (21 strains from the GRACE collection and 22 strains from the GRACEv2 collection) that were not predicted or assessed for essentiality in previously published models ${ }^{18}$, highlighting the comprehensive nature of our analyses.

Finally, our comprehensive analysis enabled the discovery of additional essential genes. Specifically, when defining known essential genes as the $1278 \mathrm{C}$. albicans genes currently marked as inviable in the Candida Genome Database ${ }^{21}$, our set of 621 experimentally confirmed essential genes from the GRACE and GRACEv2 collections included 53 essential genes that were not previously classified as essential. In terms of our essential gene predictions, at a stringent confidence cutoff (RF score $>0.5$, estimated precision $>90 \%$ ), our model covered $46 \%$ of the known essential genes and yielded 66 predicted essential genes that were not previously reported as essential. At a more relaxed, but still high confidence cutoff (RF score $>0.15$, estimated precision $>$ $70 \%$ ), we covered $88 \%$ of the known essential genes and identified 478 predicted essential genes that were not previously reported (Supplementary Fig. 2d).

We utilized co-expression clustering analysis to determine whether any biological processes are enriched among essential genes in the C. albicans genome. Using a non-linear dimensionality reduction, we clustered $C$. albicans genes based on coexpression $^{22}$ (Fig. 2c) and used GO term enrichment of the genes in each cluster to assign a putative function. We then tested each cluster for enrichment of essential genes using the hypergeometric test and found enrichment at the 0.05 significance level for four clusters: translation (120 of 198 genes; $p<8 \mathrm{e}-40$ ), ncRNA processing (123 of 299 genes; $p<8 \mathrm{e}-27$ ), chromatin organization (58 of 229 genes; $p<0.02$ ), and peptide biosynthesis (34 of 122 genes; $p<0.02$ ).

Genes that are essential in fungi but not present in humans represent priority targets for antifungal drug development, as there is a reduced likelihood of off-target toxicity ${ }^{26,28}$. Of the 621 validated C. albicans essential genes, 149 lacked human homologs (Supplementary Data 4). These genes are enriched in diverse processes including organic substance biosynthetic processes, cellular component organization, gene expression, and translation (FDR $<1 \%$; Supplementary Data 3). We paired our findings with drug target databases including DrugBank ${ }^{29}$, Therapeutic Target Database $^{30}$, and PubChem ${ }^{31}$ to identify 97 compounds targeting nine of our experimentally confirmed essential or predicted essential genes, three of them lacking human orthologs (Supplementary Data 5).

Characterization of Krp1 as a member of the kinetochore. We next leveraged our essentiality predictions to characterize the role of fungal-specific essential genes that lack annotated functions. We identified those genes without predicted S. cerevisiae orthologs using the Candida Genome Database ${ }^{21}$. Of the 149 fungalspecific essential genes, only four lacked $S$. cerevisiae orthologs: C1_01070C, C1_09670C, C6_03200W, and C2_07260C (Supplementary Data 4). We initially focused our efforts on C1_01070C, which we named KRP1 (kinetochore-related protein 1) and used BLASTp and conserved domain searches to explore potential gene function. Although this gene was previously annotated in CGD as essential, it remained completely uncharacterized, motivating us to further describe its biological function. These analyses predicted that KRP1 may localize to the MIND (Mis12/ Mtw1-Nnf1-Nsl1-Dsn1) complex to regulate chromosome segregation and cell cycle progression. Notably, no C. albicans Dsn1 homolog has been identified to date ${ }^{21}$. In S. cerevisiae, the MIND kinetochore subcomplex binds to DNA and recruits remaining assembly complexes, including the DASH/Dam1 complex, which connects microtubules with centromeres during cell division ${ }^{32}$. Although members of the DASH/Dam1 complex localize to kinetochores and regulate proper mitotic chromosome segregation in C. albicans ${ }^{33}$, a complete characterization of all ten predicted complex members has yet to be achieved.

To characterize the function of the C. albicans DASH/Dam1 complex, MIND complex, and Krp1, we examined essentiality of all known components using spot dilution assays. This was of particular interest, because although in S. cerevisiae all members of the DASH/Dam1 and MIND complexes are essential, they are all dispensable for growth in $S$. pombe $e^{34-37}$. Consistent with 
a

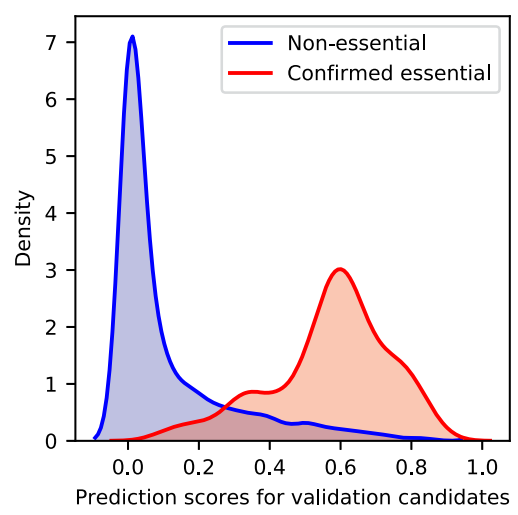

b

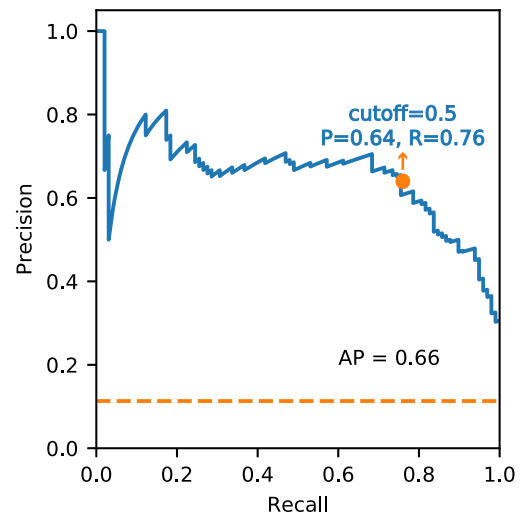

C
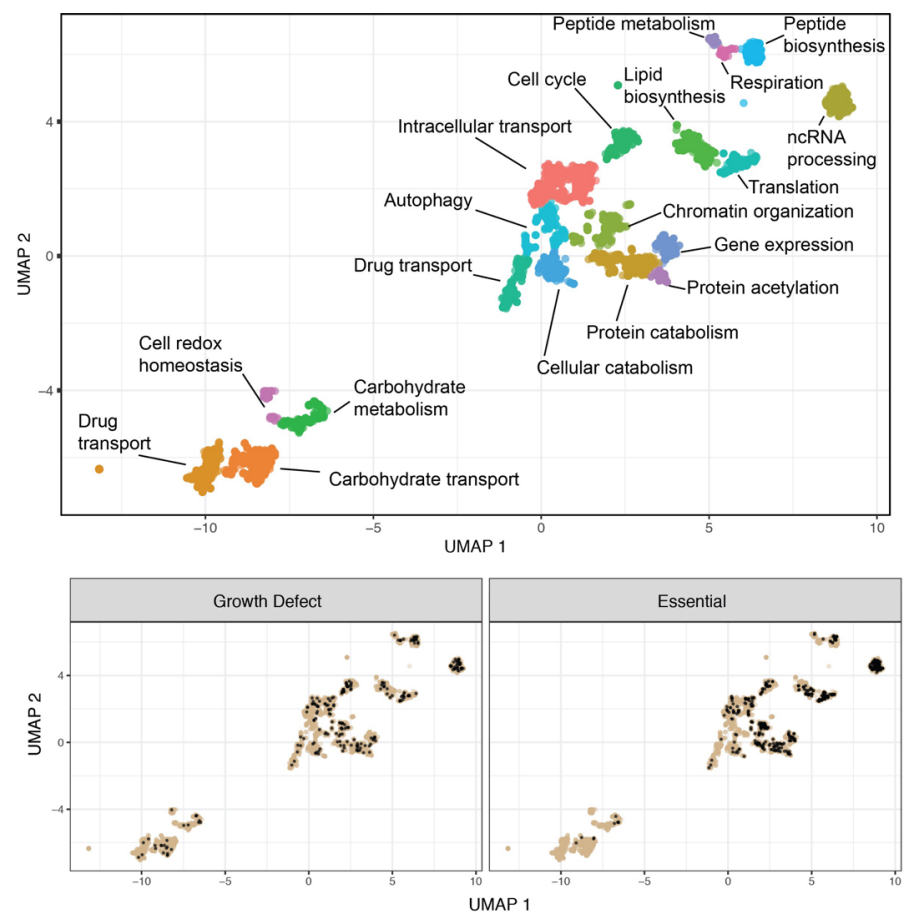

Fig. 2 Testing the accuracy of the prediction model with the GRACEv2 collection. a Distribution of prediction scores for the 98 experimentally confirmed essential genes and 768 non-essential genes from the validation candidates (GRACEv2 strains). b Precision-recall curve of the random forest model derived from the whole GRACE set and tested on the GRACEv2 experimental validation set. The default stringent cutoff score for essential gene predictions results in a precision of 0.64 and a recall of 0.76 , with an average precision score of 0.66 . c Essential genes are enriched in specific functional clusters. Clusters were generated by UMAP embedding of co-expression and functional enrichment was determined by GO term analysis. Source data are provided as a Source Data file.

previous studies in C. albicans $^{33,38}$, we found that the DASH/ Dam1 complex members SPC19, DAM1, DAD1, DAD2, and ASK1 were essential for growth, as well as the previously uncharacterized subunit genes DUO1 and DAD4 (Fig. 3a and Supplementary Fig. 3a). Interestingly, DAD3, HSK3, and SPC34 were non-essential, as substantial growth was observed despite transcriptional repression of the target gene with DOX (Fig. 3a and Supplementary Fig. 3). MIND complex members NNF1 and NSL1 were essential, along with KRP1, whereas depletion of MTW1 still allowed for growth on agar medium with a high concentration of DOX (Fig. 3a and Supplementary Fig. 3b). This is in contrast to previous work that showed MTW1 to be essential in C. albicans ${ }^{39}$.
We explored the impact of the DASH/Dam1 and MIND components on C. albicans morphology, as perturbation of the cell cycle often induces filamentation ${ }^{40,41}$, and proper function of the kinetochore is required for $G_{2} / M$ progression ${ }^{33}$. Transcriptional repression of all subunits of the DASH/Dam1 complex with a low concentration $(0.05 \mu \mathrm{g} / \mathrm{mL})$ of DOX induced filamentation in the absence of an inducing cue without significantly impacting growth, similar to the cell cycle inhibitor hydroxyurea (Fig. 3b). Similarly, the depletion of MIND complex members MTW1, NSL1, and NNF1 along with KRP1 induced filamentation (Fig. 3b).

To test whether Krp1 may function at the kinetochore, we monitored its localization using fluorescence microscopy. Both 

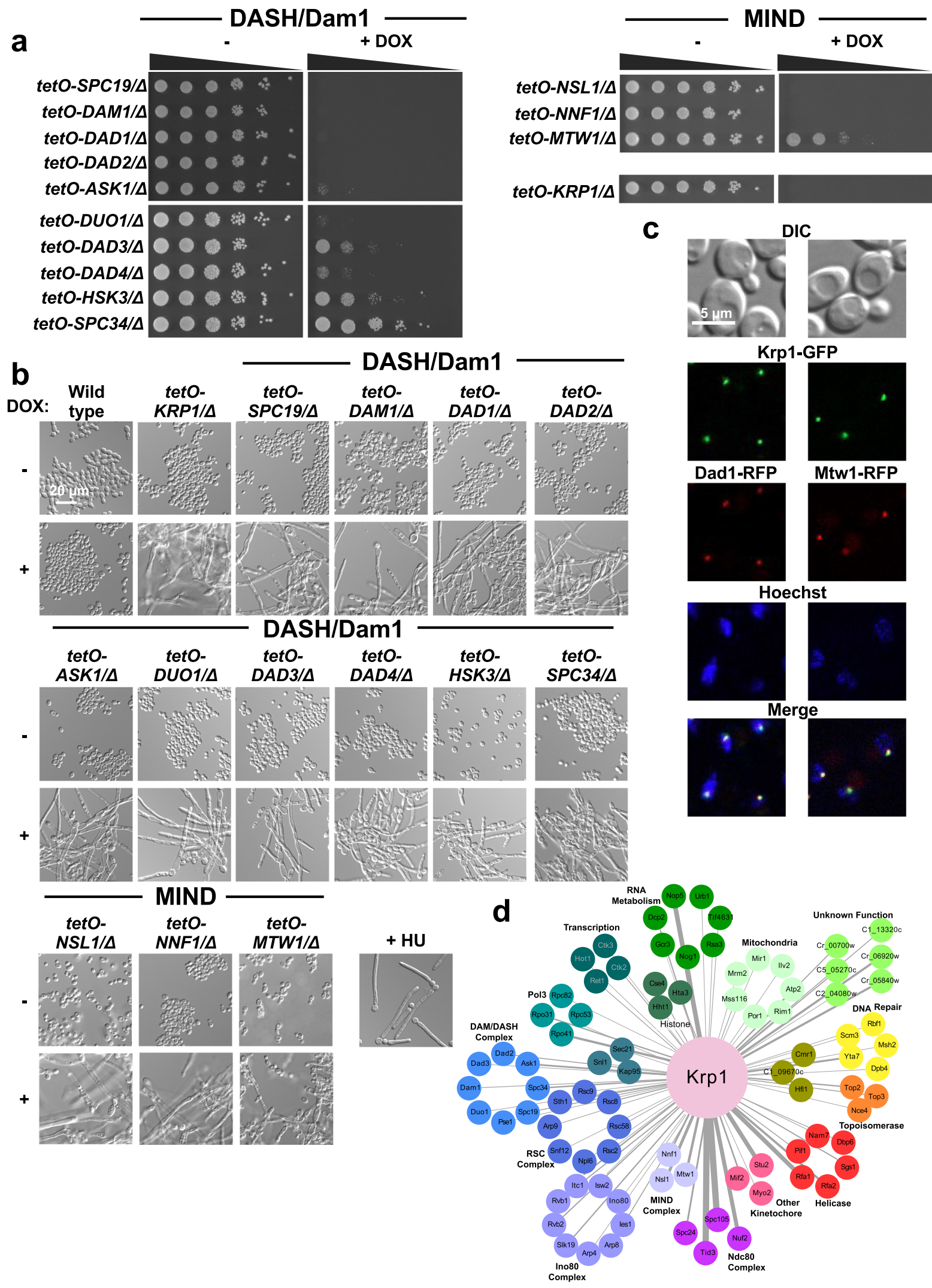

alleles of KRP1 were C-terminally tagged with a GFP epitope in a strain with both alleles of either a DASH/Dam1 complex member or MIND complex member C-terminally tagged with RFP (Dad1RFP or Mtw1-RFP, respectively). Tagging both alleles confirmed functionality, as strains were viable and grew as yeast under nonfilament inducing conditions (Fig. 3c). We observed green puncta at the nuclear periphery, which co-localized with red puncta in both strain backgrounds (Fig. 3c), suggesting that Krp1 may directly or indirectly interact with Dad1 and/or Mtw1. Finally, to elucidate the Krp1 interactome in C. albicans we performed affinity purification of Krp1-GFP using a GFP-trap resin, followed by mass spectrometry. To distinguish significant 
Fig. 3 Characterization of Krp1 as a member of the kinetochore complex. a Testing the essentiality of kinetochore components. Strains were grown overnight in the absence or presence of $0.05 \mu \mathrm{g} / \mathrm{mL}$ doxycycline $(D O X)$ at which point they were spotted in tenfold dilutions (starting from an $\mathrm{OD}_{600}$ of 0.5) onto YNB agar alone or supplemented with $50 \mu \mathrm{g} / \mathrm{mL}$ DOX. Plates were photographed after growth for $48 \mathrm{~h}$ at $30^{\circ} \mathrm{C}$. $\mathbf{b}$ Examining the impact of kinetochore-related genes on C. albicans morphology. Strains were grown overnight as described in (a). Strains were subsequently subcultured to an $\mathrm{OD}_{600}$ of $0.1 \mathrm{in} \mathrm{YPD} \mathrm{in} \mathrm{the} \mathrm{absence} \mathrm{or} \mathrm{presence} \mathrm{of} 0.05 \mu \mathrm{g} / \mathrm{mL} \mathrm{DOX}$ as indicated. The wild-type strain in the absence of DOX was treated with $25 \mathrm{mM}$ hydroxyurea (HU) as indicated. Cultures were incubated at $30^{\circ} \mathrm{C}$ for $24 \mathrm{~h}$ for GRACE strains or $6 \mathrm{~h}$ for $\mathrm{HU}$ treatment before cells were visualized by microscopy. Experiment was performed in biological duplicate with similar results. c Krp1 localizes to the kinetochore. Strains were subcultured to an $\mathrm{OD}_{600}$ of $0.1 \mathrm{in}$ YPD and allowed to grow for $4 \mathrm{~h}$ before visualization. Krp1 (green), Dad1 (red), Mtw1 (red), and nuclei (blue) were visualized by fluorescence microscopy. Experiment was performed in biological duplicate with similar results. d AP-MS of affinity-tagged Krp1 identified physically interacting proteins. Cells were grown in YPD at $30^{\circ} \mathrm{C}$, and statistically significant interactions were defined through SAINTexpress analysis compared with an unrelated tagged protein. Nodes are grouped and colored based on GO term annotation. The weight of the edges reflects the fold-change in peptide count of Krp1 relative to an unrelated tagged protein (Eno1) for those interacting partners with a BFDR $<1 \%$. Source data are provided as a Source Data file.

interactors from background, we compared interactors identified with Krp1-GFP with those identified with an Eno1-GFP control using SAINTexpress and a Bayesian false discovery rate (BFDR) cutoff of $1 \%$ (Fig. 3d and Supplementary Data 6). While Krp1 interacted with many proteins, there was a significant enrichment of interactions with proteins involved in chromosome organization, mitotic cell cycle process, and kinetochore assembly (Supplementary Data 3). Interactions were confirmed with eight out of ten DASH/Dam1 complex members and all members of the MIND complex (Fig. 3d and Supplementary Data 6). Thus, KRP1 encodes an essential protein that regulates cell cycle progression, likely through its involvement at the kinetochore.

Leveraging co-expression data to define the function of mitochondrial-related gene EMF1. To elucidate the function of another previously unannotated fungal-specific essential gene, we turned to C6_03200W. As observed by spot dilution assays, transcriptional repression of C6_03200W with DOX abrogated growth, confirming it as an essential gene (Fig. 4a and Supplementary Fig. 3c). The function of C6_03200W could not be predicted using BLASTp or conserved domain searches. Thus, we leveraged the $C$. albicans co-expression dataset used in our machine-learning model, as co-expression partners are predicted to have related functions ${ }^{22}$. C6_03200W co-clustered with mitochondrial genes, especially those involved in mitochondrial translation (Fig. 4b), and therefore we named this gene EMF1 (essential mitochondrial function 1). The mitochondrial localization prediction software Predotar v1.04 supported these findings ${ }^{42,43}$. To determine if EMF1 governs mitochondrial integrity, we used the mitochondrial dye MitoTracker Red to assess alterations in mitochondrial morphology upon transcriptional repression of this target gene. Depletion of EMF1 caused the mitochondria to coalesce and lose their tubular morphology, similar to what was observed with depletion of the wellcharacterized mitochondrial import gene TIM12 (Fig. 4c).

Next, we epitope-tagged both copies of EMF1 with GFP and monitored its localization. Tagging both alleles of EMF1 confirmed functionality, as strains were viable. Emf1-GFP colocalized with MitoTracker Red, forming discrete puncta in a similar pattern to what has been previously reported with the $C$. albicans mitochondrial DNA (mtDNA)-binding protein Gcf1 (Fig. $4 \mathrm{~d})^{44}$. To determine if Emf1 co-localizes with Gcf1, we C-terminally tagged both alleles of GCF1 with RFP in the strain harboring Emf1-GFP, and observed Emf1 and Gcf1 co-localized at DAPI-stained mitochondrial nucleoids, densely packed structures of mtDNA and associated proteins (Fig. 4e). Subsequent sequence analysis using the protein structure prediction program Phyre2 suggested Emf1 contains an HMG-box similar to the mitochondrial transcription factor A (TFAM), consistent with a putative role in binding mtDNA. As mtDNA-binding proteins are required to maintain mtDNA copy number ${ }^{45,46}$, we performed quantitative PCR to monitor changes in DNA abundance upon transcriptional repression of EMF1. Similar to GCF1, transcriptional repression of EMF1 with a low concentration of DOX caused a significant reduction in the mtDNAencoded gene NAD2 relative to the nuclear genes ACT1 and GPD1 (Fig. 4f). Together, this suggests Emf1 may act as a mtDNA-binding protein in C. albicans.

Tif33 is a member of the translation initiation complex. Subsequently, we turned to the essential gene C2_04370W, an essential gene that although previously unannotated possesses significant sequence similarity to many genes encoding eukaryotic translation initiation factor 3 (eIF3) subunits. While the eIF3 complex is conserved in eukaryotes, the number of conserved subunits, as well as the essentiality of subunits, varies substantially across species (Fig. 5a). For example, the S. cerevisiae eIF3 complex contains a core complex of five essential subunits (eIF3a, eIF3b, eIF3c, eIF3g, and eIF3i) and a non-essential subunit $(\mathrm{eIF} 3 \mathrm{j})^{47}$. In contrast, the fission yeast $S$. pombe, encodes five core eIF3 subunits, and five non-core subunits (eIF3d, eIF3e, eIF3f, eIF3h, and eIF3m ${ }^{48}$. While some eIF3 complex members have been predicted, the complex has yet to be investigated in $C$. albicans.

To identify all C. albicans eIF3 complex members, BLASTp was performed against the $C$. albicans genome with the $S$. cerevisiae and $S$. pombe subunits, identifying nine putative $C$. albicans eIF3 complex members. Six genes were predicted to function as members of the core complex: eIF3a (RPG1), eIF3b (PRT1), eIF3c (NIP1), eIF3g (TIF35), and eIF3i (TIF34), while the non-core eIF3 complex was predicted to consist of eIF3h (C3_07420W), eIF3j (HCR1), eIF3f (C5_02660C), and eIF3m (our gene of interest C2_04370W). In $S$. pombe the eIF3m subunit is encoded by TIF313, and therefore we renamed our gene of interest TIF33 (translation initiation factor 33). Transcriptional repression of RPG1, NIP1, TIF35, TIF34, C5_02660C, or TIF33 abolished growth, confirming their essentiality (Fig. 5b and Supplementary Fig. 3d). In contrast, HCR1 and C3_07420W were deemed to be non-essential. The essentiality of PRT1 could not be assessed due to insufficient transcriptional repression of the GRACE strain. These findings are consistent with observations that subunits associated with the core complex are highly conserved across eukaryotes and, wherever tested for viability, found to be essential, while the non-core members of the complex vary substantially both in terms of their presence and essentiality across species (Fig. 5a).

Next, we assessed if the putative eIF3 genes play a role in translation. We tested the sensitivity of heterozygous mutants to the translation inhibitor nourseothricin (NAT). This is based on the principle that modifying the expression of a compound target often alters the amount of compound required to inhibit that target ${ }^{11,49}$. Drug susceptibility analysis demonstrated that 
a

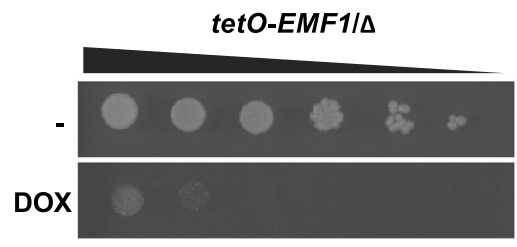

b

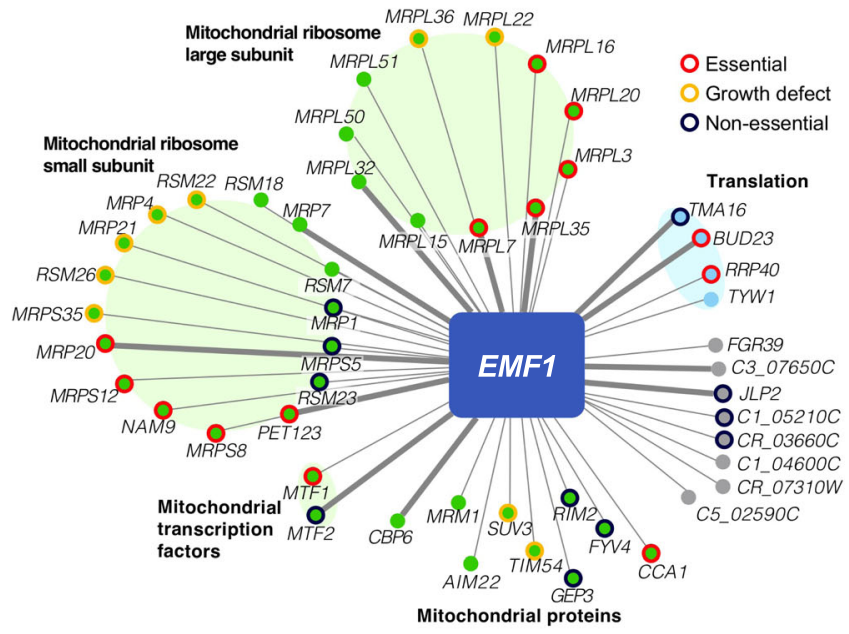

C

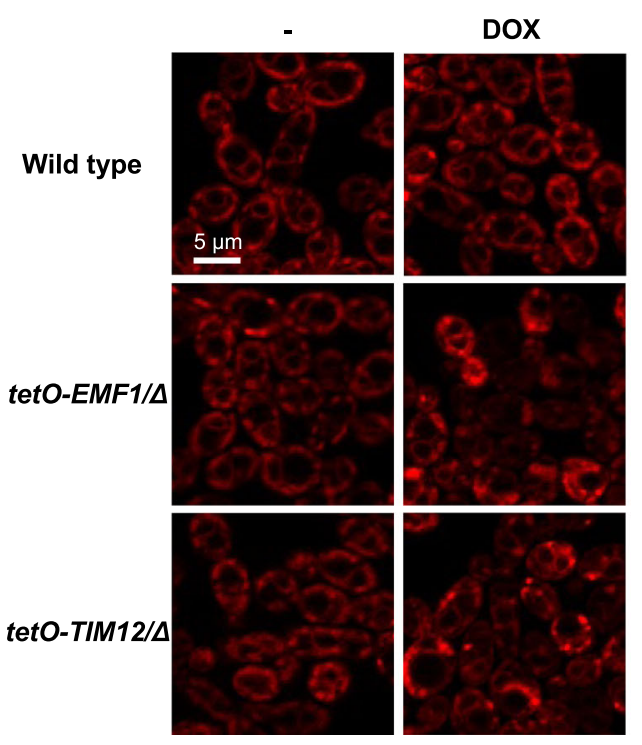

d

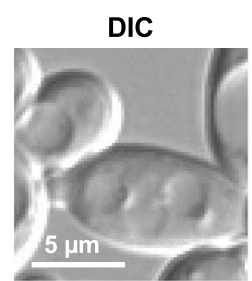

Emf1-GFP

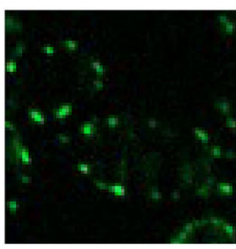

Mitotracker

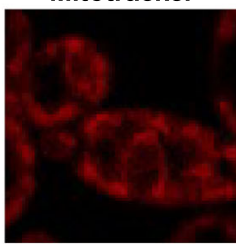

Merge

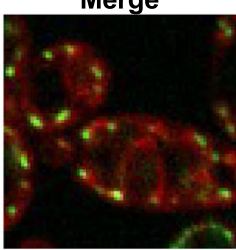

e

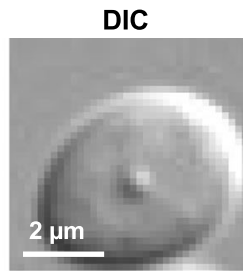

Emf1-GFP

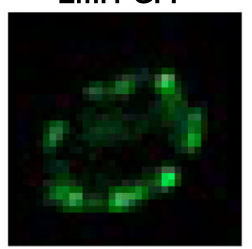

Gcf1-RFP

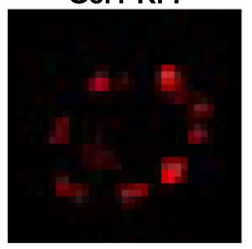

DAPI

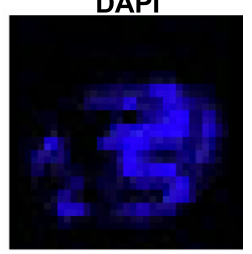

heterozygous mutants for all predicted eIF3 subunits were hypersensitive to NAT (Fig. 5c). Next, we performed a fluorescence-based translation assay. During active protein synthesis, an amino acid analog of methionine containing an alkyne moiety is incorporated into proteins. Subsequent click reaction between a green fluorescent azide and the alkyne allows for the qualitative detection of newly synthesized proteins using fluorescence microscopy and quantification by flow cytometry ${ }^{50}$. Treatment of a $C$. albicans wild-type strain with the translation inhibitor anisomycin caused a significant decrease in translation, as measured by a reduction in cellular
Merge
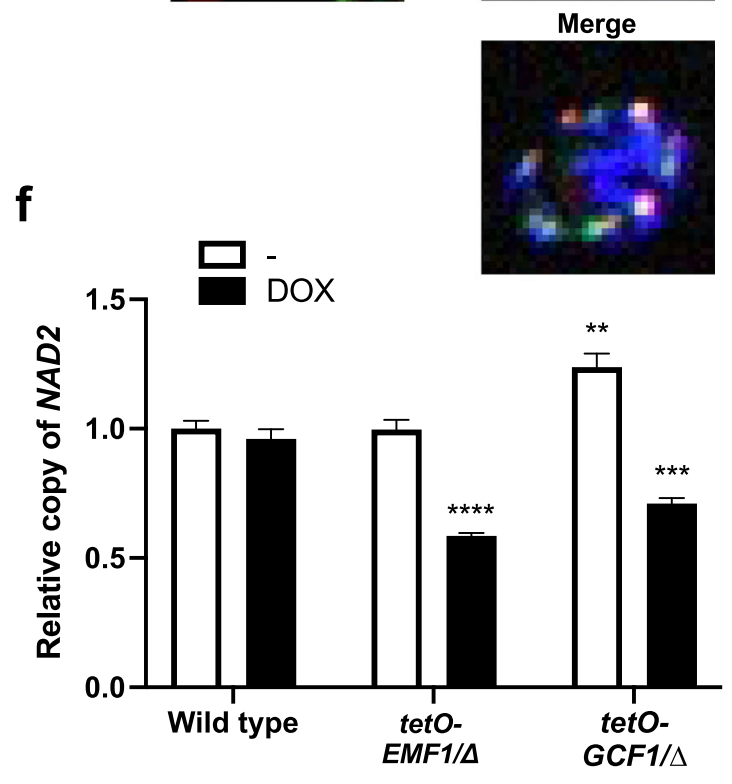

fluorescence (Fig. 5d). Depletion of all putative translation initiation subunits, including the core eIF3c subunit NIP1 and our uncharacterized gene TIF33, also caused a significant reduction in fluorescence (Fig. 5d and Supplementary Fig. 4a), indicating translation was impeded. This was specific to genes with roles in translation, as transcriptional repression of a control gene that was not involved in translation but was important for proliferation (tetO-HSP90/hsp90A) still resulted in strong fluorescence (Supplementary Fig. 4b). These results conclude Tif33 is an essential protein and a potential member of the eIF3 complex in C. albicans. 
Fig. 4 Characterization of Emf1 as a mitochondria component. a C6_03200W (renamed EMF1) is an essential C. albicans gene. The tetO-EMF1/emf14 strain was grown and assessed for essentiality as described in Fig. 3a. b A co-expression network for EMF1 identifies multiple mitochondrial proteins in the top 50 co-expressed genes. Nodes represent genes, and edges represent the strength of the co-expression. All genes had a co-expression score of at least 0.997 . Green indicates mitochondrial annotation, light blue indicates translation annotation, gray indicates no GO term annotation available. c Depletion of EMF1 perturbs mitochondrial morphology. Strains were grown overnight in the absence or presence of $0.05 \mu \mathrm{g} / \mathrm{mL} \mathrm{DOX}$, subcultured to an $\mathrm{OD}_{600}$ of 0.1 with the same respective DOX conditions, and grown for $3 \mathrm{~h}$ at $30^{\circ} \mathrm{C}$. Cultures were further incubated with $50 \mathrm{nM}$ Mitotracker Red for $40 \mathrm{~m}$, washed, and resuspended in PBS. MitoTracker Red staining was imaged with the DsRed channel with equal exposure among samples. Experiment was performed in biological duplicate with similar results. $\mathbf{d}$ Emf1 localizes to the mitochondria. Cells were subcultured to an $\mathrm{OD}_{600}$ of 0.1 and allowed to grow for $4 \mathrm{~h}$ before visualization. As indicated, cultures were incubated with $50 \mathrm{nM}$ Mitotracker Red for $40 \mathrm{~m}$, washed, and resuspended in PBS. Emf1 (green) and mitochondria (MitoTracker, red) were visualized by fluorescence microscopy. Experiment was performed in biological duplicate with similar results. e Emf1 co-localizes with Gcf1 at DAPI-stained mitochondrial nucleoids. Cells were subcultured to an $\mathrm{OD}_{600}$ of 0.1 and grown for $4 \mathrm{~h}$ before being washed and resuspended in PBS, then incubated with $1 \mu \mathrm{g} / \mathrm{mL}$ DAPI for $1 \mathrm{~h}$. Emf1 (green), Gcf1 (red), and DNA (DAPI, blue) were visualized by fluorescence microscopy. Experiment was performed in biological duplicate with similar results. $\mathbf{f}$ Transcriptional repression of EMF1 causes a significant reduction in mtDNA copy number. Relative NAD2 copy number in wild-type, tetO-EMF1/emf1A, and tetO-GCF1/gcf1 $1 \Delta$ cells in the absence and presence of $0.05 \mu \mathrm{g} / \mathrm{mL}$ DOX as determined by $\mathrm{qPCR}$, using ACT1 and GPD1 for normalization. Values shown are relative NAD2 copy number compared to the wild-type strain in the absence of DOX. Error bars represent SEM above and below the mean of technical triplicates (two-way ANOVA, Bonferroni correction for multiple comparisons, ${ }^{\star \star} P<0.002$; ${ }^{\star \star \star} P<0.0004 ;{ }^{\star \star \star \star} P<0.0001$ compared to wild-type untreated). Experiment was performed in biological duplicate with similar results. Source data are provided as a Source Data file.

Identification of an antifungal compound that targets $C$. albicans glutaminyl-tRNA synthetase. Finally, in order to investigate the therapeutic potential of compounds targeting essential genes we surveyed data from a chemical screen we performed with 9600 compounds from the University of Tokyo's Core Library that had been evaluated for single-agent activity against C. albicans. We prioritized one compound, Npyrimidinyl-beta-thiophenylacrylamide (NP-BTA), for several reasons. First, it displayed potent antifungal activity against $C$. albicans with an $\mathrm{MIC}_{80}$ of $3.125 \mu \mathrm{M}$ (Fig. 6a). Secondly, it exhibited moderate activity against the drug-resistant pathogens Candida auris and Candida glabrata (Fig. 6a). Finally, publicly available chemogenomic datasets ${ }^{51}$ suggested that NP-BTA targets glutaminyl-tRNA synthetase (GLN4) in S. cerevisiae, which we identified and confirmed as an essential gene in $C$. albicans (Supplementary Data 1 and 2).

To corroborate the $S$. cerevisiae results, haploinsufficiency (HIP) profiling was performed in C. albicans. The C. albicans double-barcoded heterozygous deletion library ${ }^{52}$ was pooled and cultured in the presence of $0.8 \mu \mathrm{M}$ NP-BTA, a concentration that inhibited growth of the pool by $\sim 20 \%$ relative to solvent-treated controls. Following high-throughput sequencing of strain-specific molecular barcodes, significantly hypersensitive hits were identified, revealing GLN4 as the heterozygous mutant with the most significant barcode reduction upon NP-BTA treatment (Fig. 6b). Hypersensitivity of this mutant to NP-BTA was confirmed relative to the heterozygous deletion collection parental strain CaSS1 (Supplementary Fig. 5a). We further assessed the impact of overexpression of GLN4 on NP-BTA sensitivity using the tetOGLN4/gln $4 \Delta$ mutant. In the absence of DOX we observed a $\sim 3$ fold increase in expression of GLN4 relative to wild type (Supplementary Fig. 5b), which resulted in a four-fold increase in the NP-BTA $\mathrm{MIC}_{80}$ (Fig. 6c). As expected, DOX-mediated repression of GLN4 sensitized C. albicans to NP-BTA (Fig. 6c).

As a complementary approach to decipher the target of NPBTA, we performed selection experiments to identify C. albicans mutants with reduced susceptibility to the compound. A $C$. albicans drug-sensitized strain was employed that contains homozygous deletions in CDR1, CDR2, MDR1, and FLU1 to enable the use of lower concentrations of compound for the selections and to reduce the likelihood of mutations that merely increase efflux. We isolated three independently evolved lineages with elevated resistance to NP-BTA that did not exhibit crossresistance to fluconazole, a compound sensitive to enhanced efflux activity (Fig. 6d and Supplementary Fig. 5c). Sanger sequencing of the putative target in these strains revealed nonsynonymous mutations in GLN4 (Fig. 6d), that mapped to the helical subdomain of the protein based on a homology model of C. albicans Gln4 that was generated based on the crystal structure of S. cerevisiae Gln4 (Fig. 6e ${ }^{53}$. The interaction between an acceptor tRNA and the helical subdomain cause conformational changes in the active site of Gln4 that permit ATP/ glutamine binding ${ }^{54}$. Computational docking of NP-BTA to $S$. cerevisiae Gln4 revealed multiple poses in which NP-BTA may bind to Gln4. The best ranked confirmation (IFD score: $-24,709.48 \mathrm{kcal} / \mathrm{mol}$ ) suggested that NP-BTA binds in the ATP/glutamine binding pocket (Fig. 6e). To further support that NP-BTA functions as a tRNA synthetase inhibitor, we assessed translation during NP-BTA treatment using the fluorescencebased translation assay. In comparison to untreated $C$. albicans cells, there was a significant reduction in fluorescence from cells treated with NP-BTA and the control translation inhibitor anisomycin (Fig. 6f), but not the control compound fluconazole, which inhibits growth but does not target translation (Supplementary Fig. 5d), strongly supporting NP-BTA as an inhibitor of Gln4.

To investigate the therapeutic potential of NP-BTA, we evaluated the efficacy of this molecule in a co-culture model, which enables assessment of both human and fungal cell proliferation in tandem ${ }^{55}$. Specifically, we used luciferase-tagged human HEK293T cells and a GFP-marked, fluconazole-tolerant C. albicans strain. Under tissue culture conditions, NP-BTA blocked C. albicans growth at $25 \mu \mathrm{M}$ (Fig. $6 \mathrm{~g}$ ). Importantly, at this concentration NP-BTA simultaneously rescued human cell survival to a comparable degree as what was observed with fluconazole (Fig. $6 \mathrm{~g}$ and Supplementary Fig. 5e). Motivated by the promising results in culture, we evaluated NP-BTA for susceptibility to hepatic inactivation by assessing its bioactivity after incubation in mouse liver microsome preparations. Technical controls included gepinacin, a compound sensitive to microsomemediated inactivation, and fluconazole, a stable antifungal ${ }^{56}$. Although fluconazole maintained antifungal activity after incubation with mouse liver microsomes for $1 \mathrm{~h}$ at $37^{\circ} \mathrm{C}$ with $5 \% \mathrm{CO}_{2}$, both gepinacin and NP-BTA lost all activity against $C$. albicans (Supplementary Fig. 5f). This precluded testing the efficacy of this compound in an in vivo model of systemic candidiasis. However, as a proof-of-principle we tested the importance of Gln4 on $C$. albicans virulence. Mice were inoculated with either wild-type or tetO-GLN4/gln $4 \Delta$ strains by retroorbital injection and supplied with drinking water supplemented with solvent or DOX to 


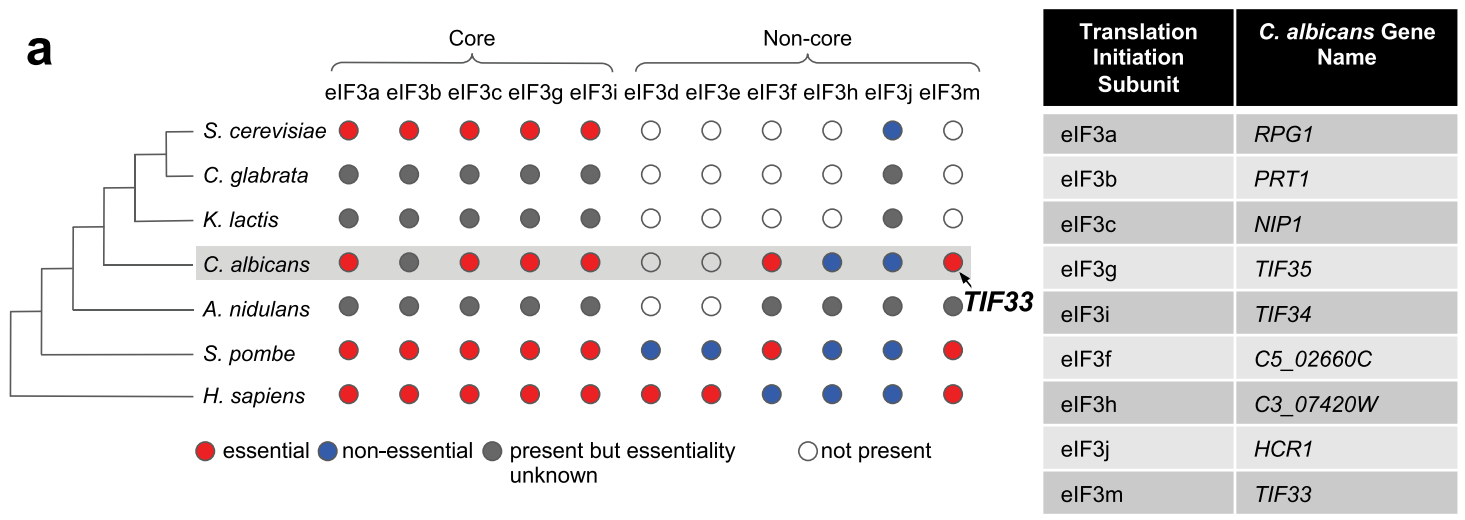

b
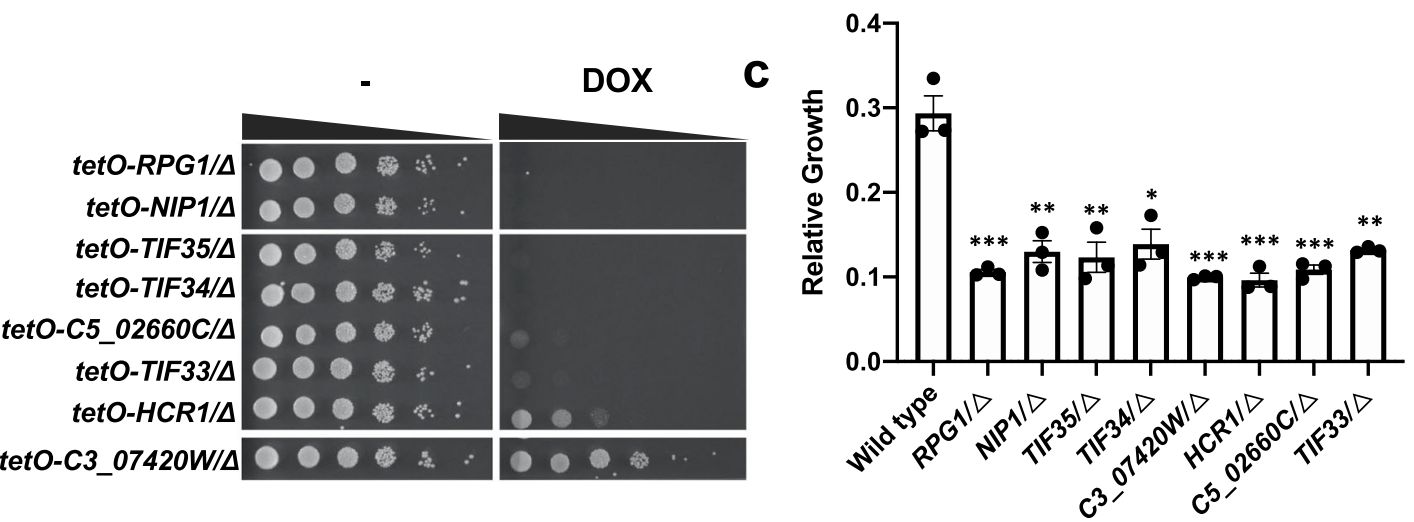

d
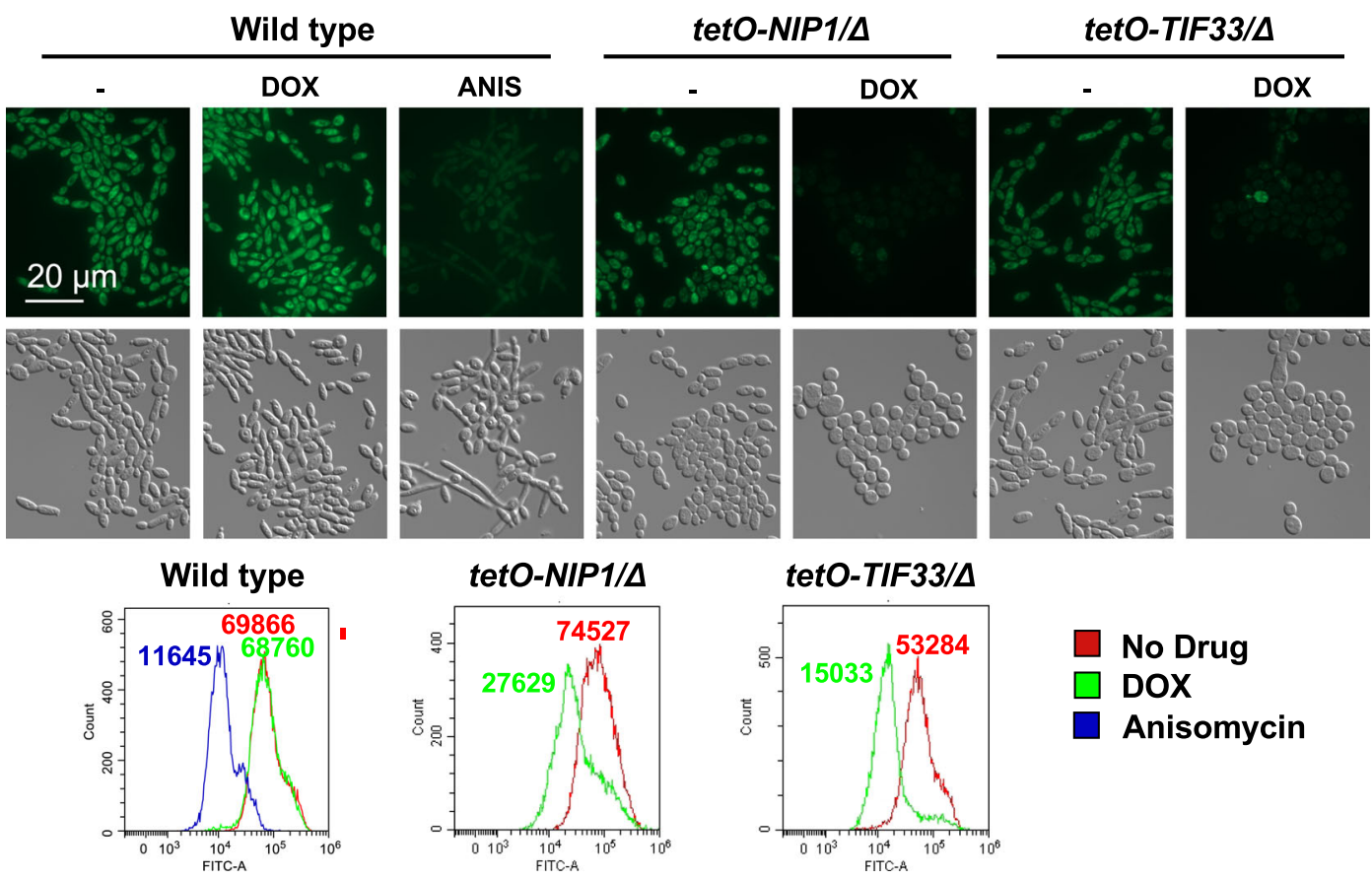

tetO-NIP1/A
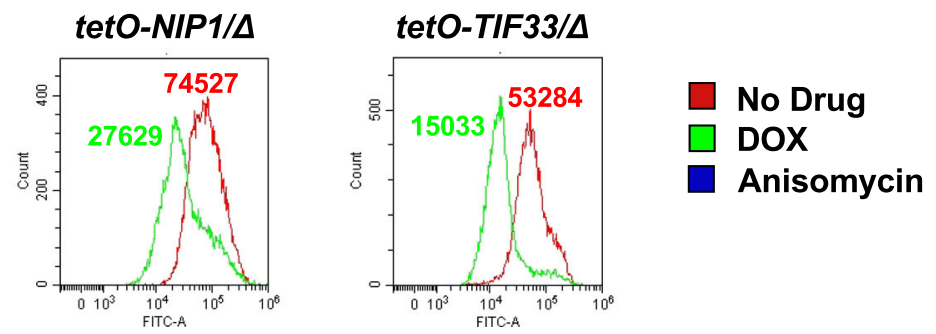

regulate the expression of GLN4 in vivo (Fig. 6h). Only those mice infected with tetO-GLN4/gln4 $\Delta$ that were provided with DOX in the drinking water saw improved survival relative to the other treatment groups, confirming GLN4 is essential for $C$. albicans virulence in vivo (Fig. $6 \mathrm{~h}, p<0.001$ ). For the three mice in the tetO-GLN4/gln4A with DOX treatment group that did succumb to the infection, this may have been due to insufficient transcriptional repression of GLN4 in vivo, the emergence of suppressor mutations in particular strains that rendered the strain resistant to DOX treatment, or other factors. Thus, our work highlights the therapeutic potential of selectively targeting the essential protein Gln4 as an antifungal strategy.

\section{Discussion}

Defining genes required for viability in pathogenic fungi not only unveils fundamental biological insights into the cellular processes that govern survival, but also reveals promising targets for therapeutic intervention. Unfortunately, genome-scale analyses of essentiality have primarily been conducted in $S$. cerevisiae with 
Fig. 5 Characterization of Tif33 as a member of the translation initiation complex. a Phylogenetic tree highlighting divergence of elF3 subunits across species. The presence of orthologs in the phylogenetic tree was derived from Wapinski et al. ${ }^{99}$, except for the $\mathrm{H}$. sapiens orthologs, which were directly identified by PomBase ${ }^{100}$. Nodes are colored based on essentiality in the indicated species. The essentiality of $C$. albicans elF3 genes was determined by our experimental test results. The essentiality of genes in S. cerevisiae and S. pombe was retrieved from Saccharomyces Genome Database ${ }^{101}$ and PomBase ${ }^{100}$, respectively. An elF3 gene in $\mathrm{H}$. sapiens was defined as essential if its CERES dependency score from the DepMap 21Q1 release ${ }^{102,103}$ was lower than -1.0 for more than $60 \%$ of the 808 CRISPR screens. b Testing the essentiality of elF3 components. Strains were grown overnight in the absence or presence of $0.05 \mu \mathrm{g} / \mathrm{mL}$ doxycycline $(\mathrm{DOX})$ at which point they were spotted in tenfold dilution (starting from an $\mathrm{OD}_{600}$ of 0.5 ) onto YNB agar alone or supplemented with $50 \mu \mathrm{g} / \mathrm{mL}$ DOX. Plates were photographed after growth for $48 \mathrm{~h}$ at $30^{\circ} \mathrm{C}$. c Heterozygous deletion mutants were grown in $\mathrm{YPD}$ at $30^{\circ} \mathrm{C}$ in the presence or absence of nourseothricin (NAT) $(8 \mu \mathrm{g} / \mathrm{mL})$. Growth was measured after $24 \mathrm{~h}$ by $\mathrm{OD}_{600}$. Average growth between technical quadruplicate wells for each strain in the presence of NAT is plotted relative to the growth of that strain in the absence of NAT. Data are presented as average values \pm SD. Significance of difference was determined by two-way ANOVA, Bonferroni correction for multiple comparisons, ${ }^{\star \star \star} P<0.001$; ${ }^{\star \star} P<0.01$; ${ }^{\star} P<0.05$. Absolute $P$ values provided in Source Data file. $\mathbf{d}$ A Click-iT protein synthesis assay kit was used to visualize protein translation. Strains were grown overnight in the absence or presence of $0.05 \mu \mathrm{g} / \mathrm{mL}$ DOX as indicated. Strains were subcultured to an $\mathrm{OD}_{600}$ of 0.1 in the same DOX conditions as the overnight and grown at $30^{\circ} \mathrm{C}$ for $4 \mathrm{~h}$. Cells were treated for $10 \mathrm{~m}$ with $100 \mu \mathrm{g} / \mathrm{mL}$ of the translation inhibitor anisomycin (ANIS), as indicated. The I-homopropargylglycine (HPG) alkyne methionine analog was added, and then the cells were fixed. The azide fluorophore was added, and cells were imaged on the GFP channel to detect if translation had occurred. Cells were analyzed by flow cytometry. Histograms depict relative fluorescence intensity (FITC-A) of a minimum 20,000 events, values depict median fluorescence intensity (MFI). Experiment was performed in biological duplicate with similar results. Source data are provided as a Source Data file.

limited studies performed directly in fungal pathogens ${ }^{18,20,57}$. Here, we developed a machine-learning model to generate the most comprehensive essentiality prediction database for a fungal pathogen to date. To test the accuracy of our predictions, we dramatically expanded coverage of the C. albicans GRACE collection by 866 strains, and experimentally confirmed 621 genes as essential under standard laboratory conditions.

We note there has been a previous effort to build a machinelearning-based model for predicting essentiality in C. albicans, based on data from a TnSeq screen ${ }^{18}$. There are two important differences in our approach. First, we used a different gene essentiality standard for training the machine-learning approach; specifically, we used the existing GRACE strains as our gold standard while the previous study used a standard derived from ortholog information. Second, we incorporated a broader set of gene features as input to the model. Specifically, Segal et al. ${ }^{18}$ used TnSeq features as input, which are indeed powerful indicators of essentiality, while we used a broader set of gene features including not only TnSeq data from Segal et al. ${ }^{18}$, but also gene expression, information from orthologs, and sequence conservation and characteristics. In detailed comparisons of our approach and the previous machine-learning model, we show that both of these differences contribute to substantial improvement in the generalizability and accuracy of predictions for gene essentiality. In particular, our updated machine-learning model provides more accurate predictions of essentiality for genes represented among the recently constructed mutants in the GRACEv2 collection (Supplementary Note 1 and Supplementary Table 1).

One notable caveat to the interpretation of essential and nonessential genes determined here is that there may be instances where the DOX-repression system results in incomplete repression of the target gene. For essential genes whose function can be fulfilled with very low expression, such cases could be falsely identified as non-essential. Based on previous observations in $S$. cerevisiae and E. coli, one would expect a higher rate of these cases among genes with a low baseline level of expression ${ }^{58}$. We did find a limited number of cases consistent with this, including the fact that our analysis deemed the lowly-expressed MIND complex member Mtw1 as non-essential, despite previous reports ${ }^{39}$. As a result, we note that the machine-learning approach used to guide strain construction has also been trained with any biases related to the experimental system as we have used the confirmed essential and non-essential GRACE strains as our training standard. This has guided us to genes for which we are likely to confirm essential using our DOX-repression system, but this potential bias should be kept in mind when interpreting predictions outside the context of our experimental system.

As an opportunistic pathogen, C. albicans colonizes anatomically diverse mucosal surfaces and it must be able to adapt to many different environmental niches to thrive. While defining the essential gene set under laboratory conditions provides a critical foundation, analyses of genes essential for growth in vivo will undoubtedly reveal additional vulnerabilities. Several C. albicans genes have already been identified that are integral for growth in a murine model of Candida infection ${ }^{59}$, as well as required for C. albicans commensalism and virulence ${ }^{17,60}$, many of which are distinct from what has been observed in vitro. Additional studies have highlighted this discordance, concluding that although select genes are essential across multiple conditions (pan-essential genes), many show variable phenotypes in distinct environmental conditions. For example, chemical genomic assays in S. cerevisiae suggested nearly the entire yeast genome (97\%) is required for optimal growth in at least one of the many distinct environmental conditions and stresses assessed ${ }^{61}$. Equally intriguing is fact that genetic background can modify gene essentiality in bacterial and fungal species ${ }^{62,63}$. This motivates continued exploration of genes required for $C$. albicans survival across different conditions to expand the antifungal target space and illuminate functional relationships in the cell.

Our analysis of gene essentiality revealed several fungal-specific essential genes with no previously annotated function. One such gene, C1_01070C or KRP1, was predicted to localize to the MIND complex, where it regulates chromosome segregation and cell cycle progression with other kinetochore-related proteins. To our knowledge, S. cerevisiae does not encode a homolog of KRP1, revealing divergent aspects of $C$. albicans biology. By coupling phenotypic characterization with a biochemical analysis to define the Krp1 interactome, we confirmed Krp1 likely functions at the C. albicans kinetochore. We also provided comprehensive characterization of all DASH/Dam1 complex members in C. albicans. The regulation of microtubule polymerization and kinetochore assembly during cell division serve as fundamental biological functions across all eukaryotes. Although many kinetochore components are evolutionarily conserved, there are divergent regulators that perform more specialized roles ${ }^{64}$. For example, the DASH/Dam1 complex is completely absent from metazoans ${ }^{64-66}$. Furthermore, although all complex members are essential in $S$. cerevisiae, they are not essential in $S$. pombe $e^{34-37}$. This speciesspecific essentiality has been attributed to differences in centromere organization and alternate kinetochore proteins ${ }^{38}$. 
a

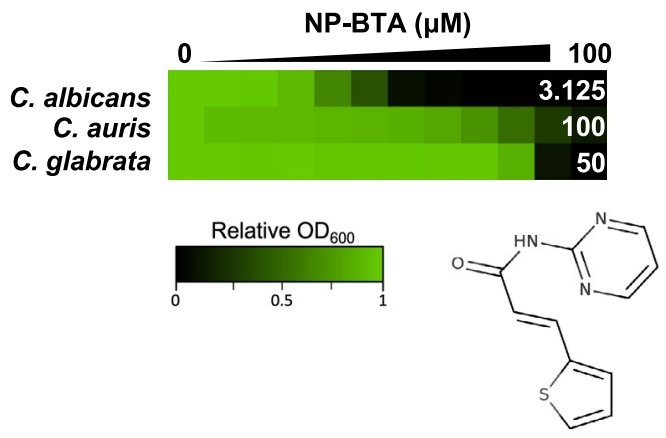

C

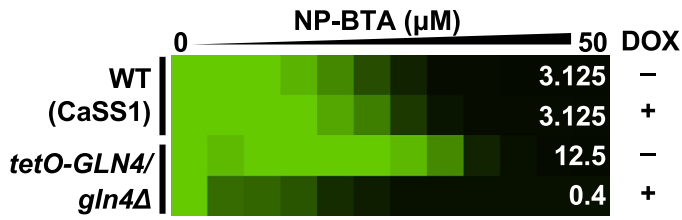

e
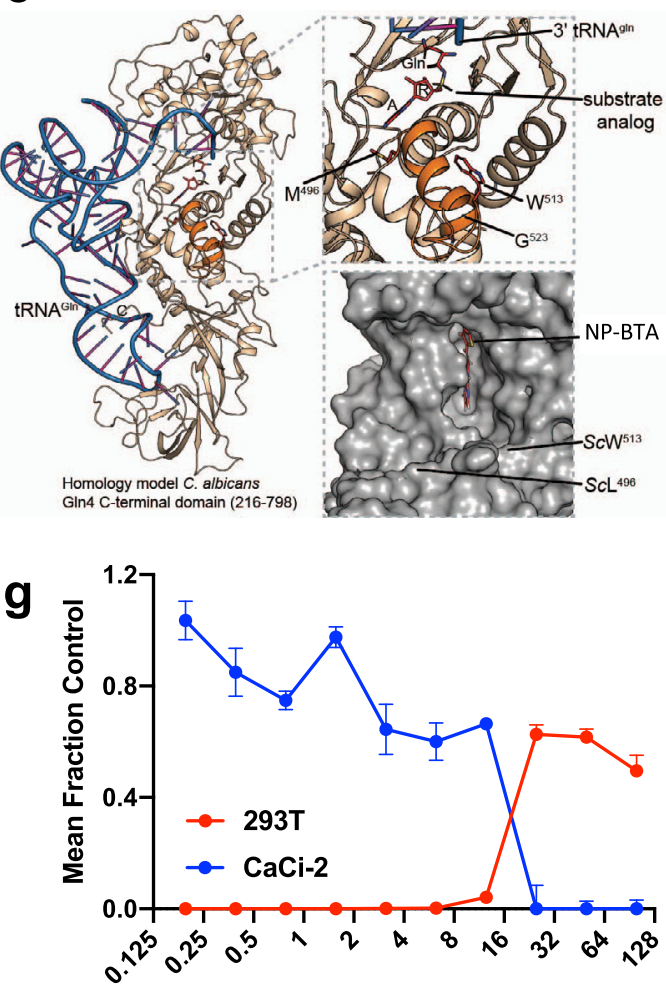

NP-BTA $(\mu \mathrm{M})$

To further define the function of fungal-specific essential genes that lack a S. cerevisiae homolog, we next turned to EMF1. Leveraging co-expression datasets 22 , we predicted it to have a role in the mitochondria. Our discovery that Emf1 localizes to the C. albicans mitochondria and is important for maintaining mitochondrial integrity illustrates the power of co-expression partners in predicting gene function, a concept that has been employed for other species ${ }^{67-70}$. Furthermore, since targeting mitochondrial proteins has emerged as a promising therapeutic approach ${ }^{59,71,72}$, characterization of this mitochondrial gene in C. albicans provides insights relevant to fundamental biology with practical applications. b

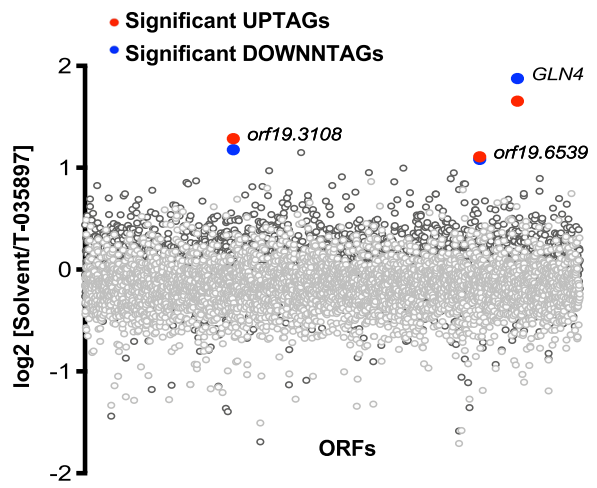

d

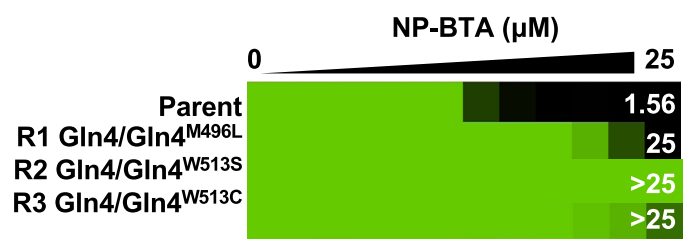

NP-BTA
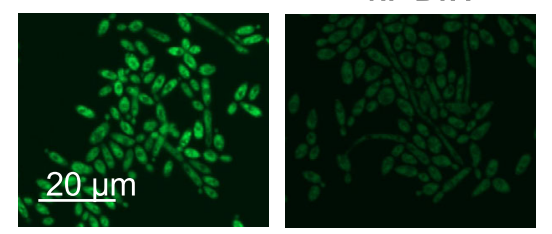

Anisomycin

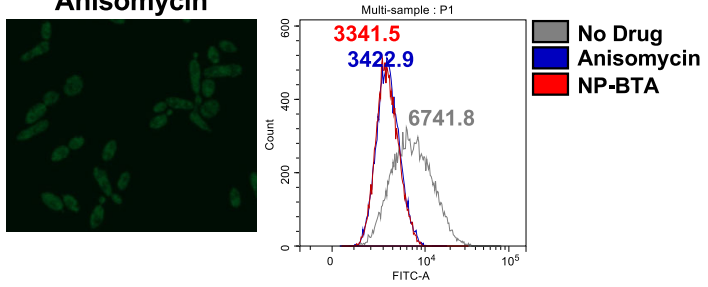

h

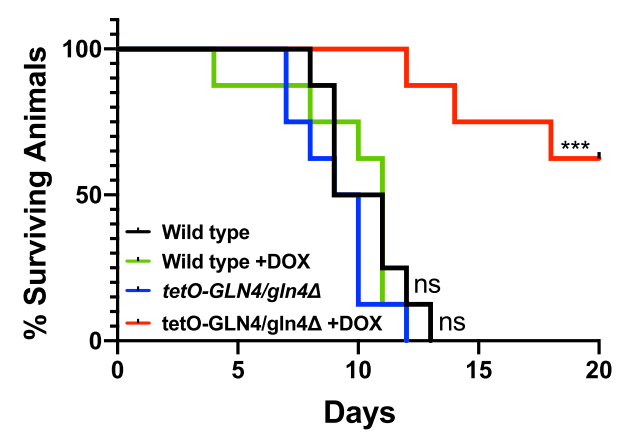

The essential genes identified in this study serve as a valuable resource to expand the antifungal drug target space. By integrating a comprehensive essentiality dataset with publicly available chemogenomic databases ${ }^{51}$, we highlight the potential of targeting the glutaminyl-tRNA synthetase Gln4 to combat $C$. albicans infections. While aminoacyl-tRNA-synthetases are essential for all organisms to attach amino acids to their cognate tRNAs for protein synthesis, we identified NP-BTA as a small molecule predicted to selectively target fungal Gln4 as it did not demonstrate significant mammalian toxicity in vitro. This is consistent with analyses demonstrating that many fungal 
Fig. 6 NP-BTA targets C. albicans glutaminyl-tRNA synthetase. a Dose-response assay based on twofold serial dilution of NP-BTA for C. albicans (SN95), C. auris (VPCI 673), or C. glabrata (F27). Assays were incubated for $24 \mathrm{~h}$ at $30^{\circ} \mathrm{C}$ in YPD and growth was normalized relative to the respective nocompound control (see color bar). $\mathrm{MIC}_{80}$ values listed in white. Structure of NP-BTA displayed below heat map. $\mathbf{b}$ The double-barcoded C. albicans heterozygous deletion collection was grown in the presence or absence of NP-BTA $(0.8 \mu \mathrm{M})$. Strains with a solvent/drug log2 ratio greater than 7 median absolute deviations (MADs) above the median were considered significant (see legend). UPTAG reads are shown in light gray, DOWNTAG reads are shown in dark gray. c Dose-response assay based on twofold serial dilution of NP-BTA for C. albicans (CaSS1) or tetO-GLN4/gln4A in the absence or presence of $0.05 \mu \mathrm{g} / \mathrm{mL}$ of doxycycline (DOX) as indicated. Assay performed as in (a). d Dose-response assay based on twofold serial dilution of NP-BTA for C. albicans parent (CaLC2749), as well as three independent resistant lineages (R1-R3). Identified Gln4 substitutions are listed. Dose-response assays were performed as in (a). e Homology model of the C-terminal domain of C. albicans Gln4 (beige, cartoon) based on the apo crystal structure of S. cerevisiae Gln4 (PDB: 4H3S; 66\% sequence identity). To illustrate the location of the active site, tRNA Gln (blue, cartoon) and glutaminyl aminoacyl-adenylate analog 5'-O-[N-(L-glutaminyl)sulphamoyl]adenosine (red spheres; A: adenosine; R: ribose; Gln: glutamine) were placed from the Escherichia coli GlnRS-tRNAsubstrate analog complex (PDB: 1QTQ), which aligned to the $C$. albicans model with an RMSD of $2.2 \AA$. Amino acids whose substitution confers reduced sensitivity to NP-BTA are shown as red sticks; numbering reflects amino acid position in C. albicans Gln4. Lower inset: Two binding poses of NP-BTA (sticks) were identified in the Gln4 active site after computational docking to the apo structure of S. cerevisiae Gln4 (gray surface). $\mathbf{f}$ Protein translation was evaluated as in Fig. 5. Cells were treated for $10 \mathrm{~m}$ with $100 \mu \mathrm{g} / \mathrm{mL}$ of anisomycin or $6.25 \mu \mathrm{M}$ NP-BTA. Histograms depict relative fluorescence intensity (FITC-A) of events, values depict median fluorescence intensity (MFI). Experiment was performed in biological duplicate with similar results. $\mathbf{g}$ Relative growth/survival of human kidney-derived cells (HEK293T-luciferase, blue line) and azole-tolerant C. albicans in co-culture (CaCi-2-GFP, CaLC867, red line). Each point depicts the mean of triplicate wells. Error bars, SEM. Four-parameter curve fitting was performed in Prism v8.4. $\mathbf{h}$ Depletion of GLN4 by the addition of DOX to the drinking water significantly improved survival relative to other conditions. Log-rank (Mantel-Cox) test, ${ }^{\star \star \star} P<0.0001$. Log-rank test for trend, $P=0.0003$. Source data are provided as a Source Data file.

aminoacyl-tRNA synthetases possess key structural differences from their mammalian homologs ${ }^{73}$. In addition, targeting aminoacyl-tRNA synthetases has been explored to treat infections caused by diverse bacteria and parasites, with the benzoxaborole compound tavaborole being the first aminoacyl-tRNA synthetase inhibitor approved for treatment of microbial infections ${ }^{74,75}$. As high-throughput sequencing and chemogenomic approaches continue to advance $\mathrm{e}^{11,76,77}$, our ability to rapidly identify compounds predicted to target essential gene products will dramatically improve, catalyzing discovery and development of muchneeded treatment options to combat life-threatening fungal disease.

\section{Methods \\ Fungal strains and growth conditions. Strains used in this study are listed in Supplementary Table 2. Plasmids used in this study are listed in Supplementary Table 3. Archives of these strains were maintained in $25 \%$ glycerol at $-80{ }^{\circ} \mathrm{C}$. Active cultures were maintained on solid yeast extract peptone (YPD; $1 \%$ yeast extract, $2 \%$ bactopeptone, $2 \%$ glucose, and $2 \%$ agar) at $4{ }^{\circ} \mathrm{C}$ for no longer than 1 month. Liquid cultures were grown in YPD at $30^{\circ} \mathrm{C}$, unless otherwise indicated. To select for nourseothricin (NAT)-resistant mutants, NAT (Jena Bioscience, 96736-11-7) was solubilized in water and supplemented into YPD agar plates $(150-250 \mu \mathrm{g} / \mathrm{mL})$. Prototrophic colonies were selected on SD plates $(0.674 \%$ yeast nitrogen base without amino acids with ammonium sulfate, $2 \%$ glucose, and $2 \%$ agar). Essentiality was tested on YNB plates $(0.17 \%$ yeast nitrogen base without amino acids and without ammonium sulfate, with $2 \%$ glucose, $0.1 \%$ glutamic acid (MSG), and $2 \%$ agar).}

Building a machine-learning model for gene essentiality prediction. To identify essential and non-essential genes in C. albicans, we constructed a random forest (RF) classifier ${ }^{25}$ using the scikit-learn library ${ }^{78}$ (version 0.23 .2 ) with the 13 features collected for 6638 C. albicans genes. We note that this gene set includes 6416 genes defined based on the A haplotype, 197 genes for which both A and B haplotype versions exist, and 5 genes that were unique to the $\mathrm{B}$ haplotype. In addition, there are 20 genes encoded on the mitochondrial genome. Details about the features used as input for our supervised machine-learning approach are in Supplementary Data 1. For genes with missing data for individual features, we imputed values using the mean of the corresponding attribute before training the RF classifier. Selected hyperparameters including the number of trees in the forest, the function to measure the quality of a split, the maximum depth of the tree, the number of features to consider for the best split, and the number of samples to draw from the training set, were optimized using fivefold cross-validation and a grid-search over a parameter grid. $80 \%$ of the 2327 genes in the C. albicans GRACE library were randomly selected for the training set, on which we conducted the fivefold crossvalidation with grid-search. The performance on the remaining $20 \%$ of the GRACE gene set using the optimal classifier derived from the $80 \%$ GRACE training set is shown in Supplementary Fig. 2b.
Experimental evaluation of the performance of model predictions. A set of 866 recently generated GRACE strains with a range of RF prediction scores were constructed for experimental validation. RF prediction scores for these genes were derived from a model trained on the complete GRACE gene set (all 2327 genes) with the previously determined optimal hyperparameters from the cross-validation described above. Prediction performance on this set was evaluated by performing receiver operating characteristic (ROC) and precision-recall analysis on the RF scores with respect to the experimentally determined essentiality verdict (see details in the "Methods" section "Testing for essentiality"). These predictions achieved an AP of 0.66 and AUC of 0.95 on this experimental validation set (Fig. $2 b$ and Supplementary Fig. 2c). Permutation feature importance, which is defined to be the decrease in a model score when a single feature value is randomly permuted ${ }^{25}$, was evaluated on the GRACE gene set with 30 repeats and reported by scikit-learn.

Mutant construction. Double-barcoded heterozygous (HET) mutants that were not available in the original collection were constructed, as described previously with some modifications ${ }^{19,52}$. The CaHIS3 cassette was PCR amplified from pLC1251 using primers containing 43 base pairs of homology to the $5^{\prime}$ or $3^{\prime}$ of the target gene, internal unique 20 base pair strain-identifying barcodes with flanking common primer sequences, and 18 base pairs which anneal to the $5^{\prime}$ or $3^{\prime}$ of the $\mathrm{CaHIS} 3$ gene (designated primer 5 and primer 6 ). The cassette was transformed into the parental strain for the library (CaLC6106), which contained the tetracycline-repressible transactivator and was auxotrophic for histidine. Prototrophic colonies were PCR tested for upstream and downstream integration using primer 3 or primer 7 together with a primer that anneals within the HIS 3 cassette (oLC6701 for primer 3 or oLC8017 for primer 7). Primer sequences used in this study are provided in Supplementary Data 7.

GRACE strains were generated from HET mutants, as described previously with some modifications ${ }^{19}$. Briefly, the NAT cassette and tet $O$ promoter were PCR amplified from pLC763 using primers containing 20-22 base pairs complementary to the promoter replacement cassette and 70 base pairs of homology upstream or downstream of the start of the gene of interest (designated primer 1 and primer 2). The cassette was transformed into the relevant heterozygous mutant. NATresistant colonies were PCR tested for upstream integration of the tet $O$ promoter using a primer that anneals $\sim 250-350$ base pairs upstream of the gene of interest (designated primer 3) and a primer that anneals within the NAT cassette (oLC6853), and/or downstream integration using a primer that anneals $\sim 250-300$ base pairs downstream of the start codon (designated primer 4) and a primer that anneals within the tet $O$ cassette (oLC2535). NAT-resistant colonies were additionally tested by PCR to verify the absence of a wild-type promoter upstream of a wild-type allele of the target gene using primer 3 and primer 4 .

CaLC7321. This strain with both copies of C6_03200W or EMF1 C-terminally tagged with GFP was made using a transient CRISPR approach adapted from Min et al. ${ }^{79}$. The GFP-NAT cassette was PCR amplified from pLC389 using oLC9284 and oLC9285. The CaCAS9 cassette was amplified from pLC963 using oLC6924 and oLC6925. The sgRNA fusion cassette was PCR amplified from pLC963 with oLC5978 and oLC9288 (fragment A) and oLC5980 and oLC9289 (fragment B), and fusion PCR was performed on fragments A and B using the nested primers oLC5979 and oLC5981. The GFP-NAT cassette, sgRNA, and Cas9 DNA were transformed into CaLC239. Upstream integration was PCR tested using oLC600 
and oLC9286, and downstream integration was tested using oLC274 and oLC9287. Lack of a wild-type allele was PCR tested using oLC9290 and oLC9286.

CaLC7334. This strain with both copies of C1_01070C or KRP1 C-terminally tagged with GFP was made using a transient CRISPR approach adapted from Min et al. ${ }^{79}$. The GFP-NAT cassette was PCR amplified from pLC389 using oLC9334 and oLC9335. The CaCAS9 cassette was amplified from pLC963 using oLC6924 and oLC6925. The sgRNA fusion cassette was PCR amplified from pLC963 with oLC5978 and oLC9338 (fragment A) and oLC5980 and oLC9339 (fragment B), and fusion PCR was performed on fragments A and B using the nested primers oLC5979 and oLC5981. The GFP-NAT cassette, sgRNA, and Cas9 DNA were transformed into CaLC239. Upstream integration was PCR tested using oLC600 and oLC9336, and downstream integration was tested using oLC274 and oLC9337. Lack of a wild-type allele was PCR tested using oLC9337 and oLC9340.

CaLC7408. This strain with both copies of C6_03200W or EMF1 C-terminally tagged with GFP and both copies and GCF1 C-terminally tagged with RFP was made using a transient CRISPR approach adapted from Min et al. ${ }^{79}$. The RFP-ARG cassette was PCR amplified from pLC1208 using oLC9381 and oLC9382. The CaCAS9 cassette was amplified from pLC963 using oLC6924 and oLC6925. The sgRNA fusion cassette was PCR amplified from pLC963 with oLC5978 and oLC9385 (fragment A) and oLC5980 and oLC9386 (fragment B), and fusion PCR was performed on fragments A and B using the nested primers oLC5979 and oLC5981. The RFP-ARG cassette, sgRNA, and Cas9 DNA were transformed into CaL7321. Upstream integration was PCR tested using oLC6971 and oLC9383, and downstream integration was tested using oLC6970 and oLC9384. Lack of a wildtype allele was PCR tested using oLC9387 and oLC9384.

CaLC7410. This strain with both copies of C1_01070C or KRP1 C-terminally tagged with GFP and both copies and MTW1 C-terminally tagged with RFP was made using a transient CRISPR approach adapted from Min et al. ${ }^{79}$. The RFP-ARG cassette was PCR amplified from pLC1208 using oLC9374 and oLC9375. The CaCAS9 cassette was amplified from pLC963 using oLC6924 and oLC6925. The sgRNA fusion cassette was PCR amplified from pLC963 with oLC5978 and oLC9378 (fragment A) and oLC5980 and oLC9379 (fragment B), and fusion PCR was performed on fragments A and B using the nested primers oLC5979 and oLC5981. The RFP-ARG cassette, sgRNA, and Cas9 DNA were transformed into CaLC7334. Upstream integration was PCR tested using oLC6971 and oLC9376, and downstream integration was tested using oLC6970 and oLC9377. Lack of a wild-type allele was PCR tested using oLC9380 and oLC9377.

CaLC7411. This strain with both copies of C1_01070C or KRP1 C-terminally tagged with GFP and both copies and DAD1 C-terminally tagged with RFP was made using a transient CRISPR approach adapted from Min et al. ${ }^{79}$. The RFP-ARG cassette was PCR amplified from pLC1208 using oLC9388 and oLC9389. The CaCAS9 cassette was amplified from pLC963 using oLC6924 and oLC6925. The sgRNA fusion cassette was PCR amplified from pLC963 with oLC5978 and oLC9392 (fragment A) and oLC5980 and oLC9393 (fragment B), and fusion PCR was performed on fragments A and B using the nested primers oLC5979 and oLC5981. The RFP-ARG cassette, sgRNA, and Cas9 DNA were transformed into CaLC7334. Upstream integration was PCR tested using oLC6971 and oLC9390, and downstream integration was tested using oLC6970 and oLC9391. Lack of a wild-type allele was PCR tested using oLC9394 and oLC9391.

Plasmid construction. Cloning procedures were performed following standard protocols. Plasmids used in this study are listed in Supplementary Table 3.

pLC1251. This vector is used to efficiently generate heterozygous deletion mutants using an analogous approach as to what was used to generate the original doublebarcoded heterozygous deletion collection (HET) ${ }^{52}$. CaHIS3 was amplified from genomic DNA from DBC strain 181 (orf19.1702), using primers oLC7860 and oLC7861 (which bind to priming sites U2 and D2). This amplicon and pLC1237 (pUC19) were digested with BamHI overnight, ligated, and transformed into DH5alpha. A diagnostic digest with BamHI was performed. Sequencing was performed with common primers M13 Forward and M13 Reverse and oLC6701.

pLC1208. The RFP coding sequence was amplified from pLC447 using oLC7964 and oLC7965. The PCR amplicon was digested with BamHI and AscI and inserted into pLC1100 (pFA-3HA-ARG) between the same sites.

Testing for essentiality. C. albicans GRACE strains were pinned into 96-well plates (Sarstedt) containing liquid YPD medium and grown overnight at $30^{\circ} \mathrm{C}$. The next day, cells were transferred into liquid YPD medium in the absence and presence of $100 \mu \mathrm{g} / \mathrm{mL}$ DOX and grown at $30^{\circ} \mathrm{C}$ overnight. Approximately $2 \mu \mathrm{L}$ of cultures were then transferred onto YNB agar plates in the absence and presence of $100 \mu \mathrm{g} / \mathrm{mL}$ DOX using a metal replicator and grown for 2 days at $30^{\circ} \mathrm{C}$. The plates were imaged and essentiality was scored by two independent people, using the scoring system outlined in Supplementary Fig. 1a. Genes were scored as essential only if the mutant had a severe growth defect in the presence of DOX and grew robustly in the absence of DOX. One biological replicate was performed for the original GRACE strains and essential verdicts were compared with a previous essentiality screen of the same library ${ }^{20}$. Two biological replicates were performed for the recently constructed GRACEv2 strains. Essentiality verdicts that were not consistent were retested and rescored through spot dilution assays. For spot dilution assays, strains were diluted to an $\mathrm{OD}_{600}$ equal to 0.5 and tenfold cell dilutions were performed. $3 \mu \mathrm{L}$ of diluted inoculum was plated onto YNB agar medium in the absence or presence of $50 \mu \mathrm{g} / \mathrm{mL}$ DOX. Plates were imaged after $48 \mathrm{~h}$ at $30^{\circ} \mathrm{C}$.

Data processing. To identify the $S$. cerevisiae orthologs of C. albicans essential genes, S. cerevisiae ortholog data by Candida Gene Order Browser (CGOB) was downloaded from the Candida Genome Database (CGD) website (http://www.candidagenome.org/ download/homology/orthologs/C_albicans_SC5314_S_cerevisiae_by_CGOB/) ${ }^{21}$. For genes without predicted orthologs in S. cerevisiae, the best hits from other species were explored using data from BLASTp analysis available on the CGD website (http:// www.candidagenome.org/download/homology/best_hits/C_albicans_SC5314_S cerevisiae_best_hits.txt). Gene Ontology (GO) was identified using the CGD Gene Ontology finder. Conserved Domain Searches were performed using the NCBI Conserved Domain Search (https://www.ncbi.nlm.nih.gov/Structure/cdd/wrpsb.cgi) ${ }^{80}$ Protein Basic Local Alignment Search Tool (BLAST) searches were performed on the NCBI website (https://blast.ncbi.nlm.nih.gov/Blast.cgi?PAGE $=$ Proteins $)^{81,82}$, on the FungiDB website (fungidb.org) ${ }^{83}$, and on the CGD website.

Identification of fungal-specific genes without human orthologs. To identify fungal-specific $C$. albicans genes among our 621 validated essential genes, we utilized data from the public orthology databases MetaPhOrs ${ }^{84}$ (version 2.0) and InParanoid $^{85}$ (version 8.0). Orthologs were identified using the default recommended cutoffs of 0.5 for Orthology Consistency Score and 1 for Evidence Level in MetaPhOrs, as well as with bootstrap support in InParanoid.

BLASTp analysis to assign gene function. All DASH/Dam1 complex members were previously identified ${ }^{21,65}$ and their identity was confirmed with BLASTp analysis. For MIND complex members, genes were annotated based on their $S$. cerevisiae homologs: NSL1 (C7_01830W), MTW1 (C2_09840W), and NNF1 (CR_01360W). No C. albicans ortholog could be identified for DNS1. To identify all the $C$. albicans eIF3 complex members, BLASTp searches were performed against the $S$. cerevisiae and $S$. pombe subunits, identifying 11 putative C. albicans eIF3 complex members. Six genes were predicted to be associated with the core complex: eIF3a (RPG1), eIF3b (PRT1), eIF3c (NIP1), eIF3g (TIF35), and eIF3i (TIF34). The non-core eIF3 complex was predicted to consist of eIF3h (C3_07420W), eIF3j (HCR1), eIF3f (C5_02660C) and eIF3m (our gene of interest $\bar{C} 2 \_04370 \mathrm{~W}$ or TIF33).

Co-expression UMAP cluster analysis. 853 bulk RNAseq runs from 18 large-scale studies of $C$. albicans available through the NCBI sequence read archive were aggregated $^{22}$. Gene $\log ($ FPKM +1$)$ expression vectors were reduced to two dimensions and clustered using Monocle $3^{86}$ by pre-processing with PCA to 500 dimensions, reducing dimensions using the UMAP algorithm ${ }^{87}$ with the following non-default parameters $\left(a=50, b=0.5, \mathrm{n} \_\right.$neighbors $=30$, negative_sample_rate $=50$, repulsion_strength $=3, \mathrm{n} \_$epochs $=2000$, and metric $=$ 'cosine'), and then clustered using the Leiden network modularity algorithm ${ }^{88}$ with non-default parameters $(k=30$, resolution $1 \mathrm{e}-5$, num_iter $=10)$. The genes in each cluster were run through the CGD GO term enrichment tool to identify potential gene function. Gene UMAP coordinates, gene clusters, and the leading gene-function signature were plotted using ggplot $2^{89}$.

Morphogenesis assays. Overnight cultures of the indicated strains were grown in YPD at $30^{\circ} \mathrm{C}$, shaking, and subcultured to an $\mathrm{OD}_{600}$ of 0.1 in YPD in the absence and presence of $0.1 \mu \mathrm{g} / \mathrm{mL}$ DOX. Cultures were grown at $30^{\circ} \mathrm{C}$ overnight before imaging on a Zeiss Axio Imager.MI.

Reverse transcriptase quantitative PCR (RT-qPCR). YPD overnights of the wild-type strain and the GRACE mutants were subcultured to an $\mathrm{OD}_{600}$ of 0.1 in YPD in the absence and presence of $0.05 \mu \mathrm{g} / \mathrm{mL}$ DOX for mitochondrial-related genes, the putative eIF3 subunit and GLN4 GRACE strains, and $0.1 \mu \mathrm{g} / \mathrm{mL}$ DOX for the Dam1/DASH and MIND complex GRACE strains. Cultures were grown overnight at $30^{\circ} \mathrm{C}$, shaking. The next day, the cultures were subcultured again to an $\mathrm{OD}_{600}$ of 0.1 in YPD in the absence and presence of the same concentration as above. Cultures were grown at $30^{\circ} \mathrm{C}$, shaking for $\sim 4 \mathrm{~h}$ or until in mid-log phase. Cells were pelleted, washed once with cold $1 \times$ phosphate buffered saline (PBS), flash-frozen, and stored at $-80^{\circ} \mathrm{C}$. Cells were lysed by bead beating four times for $30 \mathrm{~s}$, with 1 min on ice between. RNA was extracted using the QIAGEN RNeasy kit and DNase treated using the QIAGEN RNase free DNase Set. cDNA synthesis was preformed using the iScript cDNA synthesis Kit (BioRad). qRT-PCR was performed in technical triplicate with a $10 \mu \mathrm{L}$ reaction volume in a 384 -well plate, using Fast SYBR Green Master Mix (Applied Biosystems) and the BioRad CFX-384 Real Time System. The following cycling conditions were used: $95^{\circ} \mathrm{C}$ for $3 \mathrm{~min}$, 
then $95^{\circ} \mathrm{C}$ for $10 \mathrm{~s}$ and $60^{\circ} \mathrm{C}$ for $30 \mathrm{~s}$, for 40 cycles. The melt curve was completed with the following cycle conditions: $95^{\circ} \mathrm{C}$ for $10 \mathrm{~s}$ and $65^{\circ} \mathrm{C}$ for $10 \mathrm{~s}$ with an increase of $0.5^{\circ} \mathrm{C}$ per cycle up to $95^{\circ} \mathrm{C}$. The primers used to monitor expression are listed in Supplementary Data 7.

Fluorescence microscopy. Overnights of wild-type, tetO-TIM12/tim12 $\Delta$, and tetO-EMF1/emf1L strains were grown in YPD in the absence and presence of $0.05 \mu \mathrm{g} / \mathrm{mL}$ DOX at $30^{\circ} \mathrm{C}$ with shaking. Cells were subcultured to an $\mathrm{OD}_{600}$ of 0.1 in YPD in the same DOX conditions and grown for $3 \mathrm{~h}$ at $30^{\circ} \mathrm{C}$ with shaking. Cultures were further incubated with $50 \mathrm{nM}$ Mitotracker Red for $40 \mathrm{~min}$, then washed and resuspended in PBS. Fluorescent images with the DsRed channel (Mitotracker Red) were taken on a Zeiss Axio Observer.Z1 with Zeiss ApoTome Attachment, with exposure time remaining constant among samples.

For co-localization experiments, overnights for strains CaLC7321 (EMF1-GFP/ EMF1-GFP), CaLC7408 (EMF1-GFP/EMF1-GFP GCF1-RFP/GCF1-RFP), CaLC7410 (KRP1-GFP/KRP1-GFP MTW1-RFP/MTW1-RFP, CaLC7411 (KRP1GFP/KRP1-GFP DAD1-RFP/DAD1-RFP) were grown in YPD at $30^{\circ} \mathrm{C}$ with shaking. All strains were subcultured to an $\mathrm{OD}_{600}$ of 0.1 in YPD and grown for $4 \mathrm{~h}$ at $30^{\circ} \mathrm{C}$ with shaking. The CaLC7321 subculture was treated with Mitotracker Red as above. The CaLC7408 subculture was washed and resuspended in PBS, then incubated with $1 \mu \mathrm{g} / \mathrm{mL} 4^{\prime}, 6$-diamidino-2-phenylindole (DAPI) for $1 \mathrm{~h}$ on a rotating platform at room temperature. The CaLC7410 and CaLC7411 subcultures were washed and resuspended in PBS then incubated with $5 \mu \mathrm{g} / \mathrm{mL}$ Hoechst 33342 for $10 \mathrm{~min}$ on a rotating platform at room temperature. Cells were imaged by differential interference (DIC) microscopy and using the GFP channel (Emfl-GFP and Krp1-GFP), DsRed channel (Mitotracker Red, Dad1-RFP, Mtw1-RFP and Gcf1-RFP), and DAPI channel (DAPI and Hoechst 33342) on a Zeiss Axio Observer.Z1 microscope with Zeiss ApoTome attachment. For all ApoTome images, five phase images were captured, and global bleaching correction was applied.

Quantifying relative NAD2 copy number. Overnights of wild-type, tetO-EMF1/ emf $1 \Delta$, and tetO-GCF1/gcf1 $\Delta$ strains were grown in YPD in the absence and presence of $0.05 \mu \mathrm{g} / \mathrm{mL}$ DOX at $30^{\circ} \mathrm{C}$ with shaking. Cells were subcultured to an $\mathrm{OD}_{600}$ of 0.1 in YPD under the same DOX and growth conditions for $8 \mathrm{~h}$ before pelleting and storage at $-80^{\circ} \mathrm{C}$. Total genomic DNA was extracted from cell pellets using the Invitrogen PureLink Genomic DNA Kit and quantified using the Invitrogen Quant-iT PicoGreen dsDNA Assay Kit. Total genomic DNA (20 ng) was analyzed by qPCR in technical triplicate with a $10 \mu \mathrm{L}$ reaction volume in a 384-well plate, using Fast SYBR Green Master Mix (Applied Biosystems) and the BioRad CFX-384 Real Time System. The following cycling conditions were used: $95^{\circ} \mathrm{C}$ for $3 \mathrm{~min}$, then $95^{\circ} \mathrm{C}$ for $10 \mathrm{~s}$ and $60^{\circ} \mathrm{C}$ for $30 \mathrm{~s}$, for 40 cycles. The melt curve was completed with the following cycle conditions: $95^{\circ} \mathrm{C}$ for $10 \mathrm{~s}$ and $65^{\circ} \mathrm{C}$ for $10 \mathrm{~s}$ with an increase of $0.5^{\circ} \mathrm{C}$ per cycle up to $95^{\circ} \mathrm{C}$. The primers used to monitor expression are listed in Supplementary Data 7. Relative NAD2 copy number was calculated by first normalizing NAD2 levels with ACT1 and GPD1 levels for each respective sample and then normalized to wild type in the absence of DOX. Experiments were performed with two biological replicates. BioRad CFX Manager (version 3.1) was used to normalize RT-qPCR data and plot mean values with calculated SEM.

Mass spectrometry. AP-MS experiments were performed as previously described ${ }^{90,91}$, with minor modifications. Briefly, the GFP-tagged Krp1 (C1_01070c) strain was grown overnight at $30^{\circ} \mathrm{C}$ in YPD. Stationary phase cultures were diluted to an $\mathrm{OD}_{600}$ of 0.1 in $1 \mathrm{~L} \mathrm{YPD}$ and grown to an $\mathrm{OD}_{600}$ between 0.6 and 0.8 . Cells were harvested at $1720 \times g$ for $30 \mathrm{~min}$ at $4{ }^{\circ} \mathrm{C}$, washed with ice-cold water, and snap-frozen in an ethanol-dry ice bath. As a control, GFP-tagged Enol was prepared in the same manner. For protein extraction, samples were diluted 1:1 by weight in lysis buffer (50 mM HEPES [pH 7.5], $150 \mathrm{mM} \mathrm{NaCl}, 5 \mathrm{mM}$ EDTA, $5 \mathrm{mM}$ DTT, $0.1 \%$ NP- $40,1 \times$ ROCHE protease inhibitor cocktail tablet [Roche Diagnostics, Mississauga, ON, Canada]) and vortexed with glass beads $(0.5 \mathrm{~mm})$ for $4 \times 1 \mathrm{~min}$. Lysates were collected by stacked transfer for $1 \mathrm{~min}$ at $100 \times g$ with a $27^{1 / 2}$-gauge needle and clarified by centrifugation at $16,110 \times g$ for $20 \mathrm{~m}$ in $4^{\circ} \mathrm{C}$ in a microcentrifuge.

To affinity purify the GFP-interacting proteins, we used the GFP trap affinity resin (ChromoTek, Martinsried, Germany). The resin was equilibrated three times with $1 \mathrm{~mL}$ of lysis buffer (50 mM HEPES [pH 7.5], $150 \mathrm{mM} \mathrm{NaCl}, 5 \mathrm{mM}$ EDTA, $5 \mathrm{mM}$ DTT, $0.1 \%$ NP-40, $1 \times$ ROCHE protease inhibitors [Roche Diagnostics, Mississauga, ON, Canada]), using $25 \mu \mathrm{L}$ resin for each $1 \mathrm{~L}$ culture. The protein extract was then added to the resin and rotated for $2 \mathrm{~h}$ at $4{ }^{\circ} \mathrm{C}$. The beads were then washed with $1 \mathrm{~mL}$ of lysis buffer and then $1 \mathrm{~mL}$ of wash buffer $(20 \mathrm{mM}$ Tris [pH $8.0], 2 \mathrm{mM} \mathrm{CaCl} 2)$. The samples were digested on-bead with $0.75 \mu \mathrm{g}$ trypsin $(0.2 \mu \mathrm{g} /$ $\mu \mathrm{L}$ in $20 \mathrm{mM}$ Tris- $\mathrm{HCl}, \mathrm{pH} 8.0$ ) at $37^{\circ} \mathrm{C}$ overnight with rotation. The beads were then magnetized, and the supernatant was transferred to a fresh tube and incubated with $0.5 \mu \mathrm{g}$ trypsin without rotation at $37^{\circ} \mathrm{C}$ for $4 \mathrm{~h}$. Formic acid was added to a final concentration of $1-2 \%$ and the samples were kept at $-80^{\circ} \mathrm{C}$ until used for MS.

AP-MS samples and controls were analyzed by MS in two biological replicates, as previously described ${ }^{90,91}$, with the following modifications. Samples in $5 \%$ formic acid were directly loaded at $800 \mathrm{nl} / \mathrm{min}$ for 20 min onto a $100 \mu \mathrm{m} \times 15 \mathrm{~cm}$ nano-spray emitter. Peptides were eluted from the column with an acetonitrile gradient generated by an Eksigent ekspert ${ }^{\mathrm{tw}}$ nanoLC 425 and analyzed on a TripleTOF ${ }^{*} 6600$ instrument (AB SCIEX, Concord, Ontario, Canada). The gradient was delivered at $400 \mathrm{~nL} / \mathrm{min}$ from $2 \%$ acetonitrile with $0.1 \%$ formic acid to $35 \%$ acetonitrile with $0.1 \%$ formic acid using a linear gradient of $90 \mathrm{~min}$. This was followed by a $15-\mathrm{min}$ wash with $80 \%$ acetonitrile with $0.1 \%$ formic acid, and equilibration for another $15 \mathrm{~min}$ to $2 \%$ acetonitrile with $0.1 \%$ formic acid. The MS1 scan had an accumulation time of $250 \mathrm{~ms}$ within a mass range of 400-1800 Da. This was followed by ten MS/MS scans of the top ten peptides, with accumulation time of $100 \mathrm{~ms}$ for each MS/MS scan. Each candidate ion was required to have a charge state from 2 to 5 and a minimum threshold of 300 counts per second, isolated using a window of $50 \mathrm{mDa}$. Previously analyzed candidate ions were dynamically excluded for $7 \mathrm{~s}$. MS data generated were stored, searched, and analyzed using the ProHits laboratory information management system (LIMS) platform ${ }^{92}$ and searched using Mascot (v2.3.02) and Comet (v2012.02 rev.0) against the C. albicans RefSeq database (version 68), as previously described ${ }^{90,91}$ The database parameters were set to search for tryptic cleavages, allowing up to two missed cleavage sites per peptide, with a mass tolerance of $35 \mathrm{ppm}$ for precursors with charges of $2+$ to $4+$ and a tolerance of $\pm 0.15 \mathrm{amu}$ for fragment ions. Variable modifications were deamidated asparagine and glutamine residues and oxidized methionine residues. The results from each search engine were analyzed through the Trans-Proteomic Pipeline (TPP v4.6 OCCUPY rev 3$)^{93}$ via the iProphet pipeline ${ }^{94}$. SAINTexpress (v3.3) ${ }^{95}$ was used as a statistical tool to calculate the probability value of each potential protein-protein interaction from background contaminants using default parameters and a ProteinProphet cutoff of 0.95 and a minimum of two unique peptides. Controls were kept uncompressed and a BFDR of $1 \%$ or lower was required for proteins to be classified as significant interaction partners. Cytoscape (v3.8.2) was used to generate Krp1 interaction network. Proteomics data were deposited in the ProteomeXchange database (PXD029002) through partner MassIVE (massive.ucds.edu; MSV000088204).

Growth curve assays. Wild-type and heterozygous deletion mutants were grown in YPD at $30^{\circ} \mathrm{C}$ overnight, shaking. Dose-response assays with NAT or NP-BTA were performed according to standard protocols ${ }^{96}$ in 96 -well or 384 -well, flatbottom microtiter plates (Sarstedt). Growth at $30^{\circ} \mathrm{C}$ was determined by measuring $\mathrm{OD}_{600}$ using a CG-12 Cell-Grower Robot, third generation (S\&P Robotics) at $24 \mathrm{~h}$ (for NAT sensitivity) or monitored over every $15 \mathrm{~min} 24 \mathrm{~h}$ (for NP-BTA). Relative growth of the mutants for NAT sensitivity was calculated by normalizing the $\mathrm{OD}_{600}$ of each mutant in $8 \mu \mathrm{g} / \mathrm{mL}$ NAT compared to growth in no drug, and was plotted relative to the normalized growth of the wild-type strain. For NP-BTA sensitivity, growth of the mutants was determined by normalizing the ratio of the area under the curve (AUC) in the presence of drug to the AUC in drug-free medium against that of the wild-type control.

Dose-response assays and cidality testing. Antifungal drug susceptibility was measured using a modified broth microdilution protocol in YPD medium and 96well plate (Sarstedt) format, as previously described ${ }^{96}$. Following $\mathrm{OD}_{600}$ measurements, cultures were stamped onto compound-free YPD agar to assess cidality. GRACE strains were tested in YPD medium in the presence or absence of $0.05 \mu \mathrm{g} /$ $\mathrm{mL}$ and $100 \mu \mathrm{g} / \mathrm{mL}$ DOX as indicated. Data were displayed quantitatively as heat maps using Java TreeView software (version 1.1.6r4). Reported results are representative of two independent experiments, each performed in technical duplicate.

Translation assay. Translation assays were performed with the Click-iT ${ }^{\circledast}$ HPG Alexa Fluor ${ }^{\circledR} 488$ Protein Synthesis Assay Kit (Thermo Fisher C10428), according to manufacturer's instructions with some modifications. Overnights of wild-type and GRACE strains were subcultured in YPD in the presence and absence of $0.05 \mu \mathrm{g} / \mathrm{mL}$ DOX and grown at $30^{\circ} \mathrm{C}$ overnight, shaking. Cells were subcultured to an $\mathrm{OD}_{600}$ of 0.2 into $10 \mathrm{~mL}$ synthetic defined (SD) medium without amino acids, without ammonium sulfate supplemented with $2 \%$ glucose, monosodium glutamate, histidine and uridine in the presence and absence of $0.05 \mu \mathrm{g} / \mathrm{mL}$ DOX and grown at $30^{\circ} \mathrm{C}$, shaking, to mid-log phase. For compound treatment, $2 \mathrm{~mL}$ aliquots of wild-type cells were spun down and the pellet was resuspended in SD with or without $100 \mu \mathrm{M}$ anisomycin or $6.25 \mu \mathrm{M}$ NP-BTA. Cultures were incubated with or without inhibitors for $10 \mathrm{~min}$ in shaking conditions at $30^{\circ} \mathrm{C}$, before adding the L-homopropargylglycine (HPG) alkyne methionine analog at a 1:1000 dilution. For the genetic depletion samples, equal amounts of cells were spun down from each of the samples and the supernatant was removed. Pellets were resuspended in $1 \mathrm{~mL}$ of SD in the presence and absence of $0.05 \mu \mathrm{g} / \mathrm{mL}$ DOX with a 1:1000 dilution of HPG. All HPG treated samples were incubated at $30^{\circ} \mathrm{C}$, shaking, for $15 \mathrm{~min}$. Cells were spun down, supernatant removed, and pellets washed once with $1 \times$ PBS before resuspended in $500 \mu \mathrm{L}$ PBS. Cells were fixed by adding $500 \mu \mathrm{L} 70 \%$ ethanol in $1 \times$ PBS and rocking for $1 \mathrm{~h}$ at room temperature. Cells were then pelleted and washed twice with 3\% BSA in PBS. Pellet was resuspended with $500 \mu \mathrm{L}$ of the reaction cocktail containing the azide fluorophore (prepared as according to the manufacturer's protocol) and incubated for 30 min, shaking in the dark. The samples were washed once with the rinse buffer, pelleted and resuspended in $75 \mu \mathrm{L}$ of $1 \times$ PBS before imaging. Cells were imaged by differential interference contrast 
microscopy and the EGFP channel on a Zeiss Axio Imager.MI at the same exposure time. To quantify the fluorescence of each sample, a CytoFlex Flow Cytometer (Beckman Coulter) was used. A 1:10 dilution of the cell suspension was prepared in PBS in a flat-bottom, transparent, 96-well plate (Beckman Coulter) to a final volume of $200 \mu \mathrm{L}$. Each sample was run using the CytExpert Software (version 2.4) until $\sim 20,000$ events had been recorded. Appropriate gating strategies were applied to all samples (Supplementary Fig. 6). Histograms show the FITC value for each event in a population following gating to exclude debris and doublets.

Haploinsufficiency profiling (HIP). Double-barcoded, heterozygous (HET) deletion mutant pools stored in $25 \%$ glycerol at $-80^{\circ} \mathrm{C}$ were diluted to $\mathrm{OD}_{600}=0.1$ in YPD and grown for $90 \mathrm{~min}$ at $30^{\circ} \mathrm{C}$, shaking. Cultures were diluted twofold into a final volume of $5 \mathrm{~mL}$ YPD in the presence or absence of $0.8 \mu \mathrm{M}$ NP-BTA (dissolved in DMSO) and grown in triplicate for $18 \mathrm{~h}$ at $30^{\circ} \mathrm{C}$, shaking. Cells were harvested by centrifugation and cell pellets were stored at $-80^{\circ} \mathrm{C}$. Genomic DNA was extracted (PureLink Genomic DNA Mini Kit, Invitrogen) and barcodes were PCR amplified from $56 \mathrm{ng}$ of genomic DNA. Universal and indexed UPTAG and DNTAG barcode primers are described in Supplementary Data 7. UPTAG and DNTAG barcode pools were combined in an equal ratio and sequenced on an Illumina NextSeq500 instrument (Mid-Output, V2 Chemistry). Sequencing primers are described in Supplementary Data 7. UPTAG and DNTAG read frequencies for each strain were compiled into separate indexed samples, representing technical triplicates for the solvent control and drug conditions. Strains whose UPTAGs or DNTAGs in the solvent control were $<20 \%$ of the median read frequency were excluded from analysis. $\log 2$ fold changes in the UPTAG and DNTAG representation for each strain were calculated. Strains were considered to be significantly reduced in abundance if the $\log 2$ fold-change in the solvent over drug condition was $>7$ median absolute deviations (MADs) above the overall median in both the UPTAG and DNTAG, or if either barcode was $>7$ MADs and the other barcode was omitted due to insufficient reads.

Resistant mutation selection experiments. The C. albicans drug-sensitized background (CaLC2749) was used to evolve three independent lineages on solid YPD plates containing $6.24 \mu \mathrm{M}$ NP-BTA. $1 \times 10^{8}$ cells were plated and incubated at $30{ }^{\circ} \mathrm{C}$ for $3-5$ days until single colonies appeared. PCR amplification of GLN4 using primers oLC9302 and oLC9303, followed by Sanger sequencing of the PCR products using primers oLC9304-oLC9311 was performed to identify resistanceconferring mutations in GLN4.

Homology modeling and computational docking. A homology model of $C$. albicans Gln 4 was generated by one-to-one threading using the Phyre2 server ${ }^{97}$, using S. cerevisiae Gln 4 as a template (PDB: $4 \mathrm{H} 3 \mathrm{~S}$ ). Computational docking of NPBTA was performed using Glide (Maestro release 2020-4; Schrödinger LLC, New York, NY, 2020 ${ }^{98}$. Using the Maestro Protein Preparation module, S. cerevisiae Gln4 was imported (PDB 43HS), waters were removed, hydrogens were added, Het states were generated (Epik; pH 7.5), H-bonds were assigned/optimized (PROPKA; $\mathrm{pH}$ 7.5), and restrained minimization was performed (Impref; heavy atoms restrained $0.3 \AA$ ). NP-BTA was imported using the LigPrep module; possible ionization states were generated (Epik; $\mathrm{pH} 7.5$ ), and all possible tautomers/stereoisomers were generated. Docking of NP-BTA was performed using the Induced Fit Docking module, which performs rigid-body docking (Glide), followed by localized minimization (Prime), and re-docking (Glide). The resultant poses were ranked using the induced fit docking score.

Co-culture assays. The HEK293T (CRL-3216) cell line was purchased from the American Type Culture Collection (ATCC) and maintained at $37^{\circ} \mathrm{C}$ and $5 \% \mathrm{CO}_{2}$ in DMEM medium (Sigma, D5796) supplemented with $10 \%$ fetal bovine serum (FBS, Gibco 16000044). Experiments were performed using cells within 15 passages post-recovery from low-passage stocks stored in liquid nitrogen, and confirmed by PCR to be negative for mycoplasma contamination. Human HEK293T cells expressing firefly luciferase were seeded at 2000 cells/well in a 384-well plate (Sarstedt) in DMEM supplemented with 10\% FBS. After adherence overnight, GFP-labeled C. albicans CaCi-2 (CaLC867) was added at 25,000 cells $/ \mathrm{mL}$ in an equal volume. Twofold dilutions of NP-BTA or fluconazole were then added to the wells and cells were incubated for $72 \mathrm{~h}$. Relative fluorescence ( $480 \mathrm{~nm}$ excitation, $540 \mathrm{~nm}$ emission) per well was measured. $5 \mu \mathrm{L}$ Steady-Glo Luciferase Assay (Promega Cat \#E2520) reagent was then added per well and incubated for $15 \mathrm{~min}$, followed by measurement of relative luminescence per well. Both fluorescence and luminescence readouts were obtained using a Tecan Spark microplate reader. Reported results are representative of two independent experiments, each performed in technical triplicate.

Microsome stability assay. Microsome stability assay was performed as described previously ${ }^{56}$. In brief, $3 \mu \mathrm{M}$ or $6 \mu \mathrm{M}$ NP-BTA was incubated in mouse liver microsomes $(0.5 \mathrm{mg} / \mathrm{mL}$ ) and an NADPH-regenerating buffer system (Xenotech L1500), or in buffer alone. $50 \mu \mathrm{M}$ fluconazole and $40 \mu \mathrm{M}$ gepinacin were included as metabolically stable and unstable control compounds, respectively. Reaction mixtures were incubated for $1 \mathrm{~h}$ at $37^{\circ} \mathrm{C}+5 \% \mathrm{CO}_{2}$. Microsomes were inactivated by addition of $1 \mathrm{mM}$ PMSF and incubated for an additional 30 min. Reaction mixtures were then diluted 1:4 in YPD medium supplemented with $1 \times$ penicillinstreptomycin containing C. albicans (SN95) inoculum at $\mathrm{OD}_{600}=0.0005$. Cultures were incubated for $24 \mathrm{~h}$ at $30^{\circ} \mathrm{C}$ and fungal growth was assessed by $\mathrm{OD}_{600}$ measurement. Reported data are representative of two independent experiments, each performed in technical quadruplicate.

Mouse model of Candida infection. For the time to illness assay, groups of 8- to 10-week-old female BALB/c mice (Charles River) were received and treated with drinking water for 1 day ( 8 mice per group, 4 mice per cage). The next day, 16 mice were given drinking water containing $5 \%$ glucose with doxycycline hyclate (final concentration $250 \mu \mathrm{g} / \mathrm{mL}$ ) (TGI:: 24390-14-5) for 7 days prior to the infection. Meanwhile, 16 mice received drinking water with $5 \%$ glucose only as a control. Glucose was included to ensure sufficient uptake of the water. Strains were grown in YEPD overnight at $30^{\circ} \mathrm{C}$. Cells were subsequently diluted to $\mathrm{OD}_{600} 0.1$ in $100 \mathrm{~mL}$ YEPD broth and incubated at $30^{\circ} \mathrm{C}$ for $4 \mathrm{~h}$ with constant shaking. Cells were collected and washed twice with $0.9 \%$ saline (Baxter:2F7123). The number of cells was counted by hemocytometer and $4 \times 10^{5}$ cells were injected retro-orbitally into each mouse. The inoculum was confirmed by plating on a YPD agar plate and CFU counts after 2 days of growth. Mice were monitored daily and euthanized when was moribund (hunched posture, minimal motor activity, or BCS of 2 or less). Rodent housing room temperature ranges of $69-74^{\circ} \mathrm{F}$ with $30-70 \%$ humidity. A $12 \mathrm{~h}$ light $/ 12 \mathrm{~h}$ dark cycle was used. Animal experiments were conducted with approval from UCSF Institutional Animal Care and Use Committee (protocol number AN189431-01).

Reporting summary. Further information on research design is available in the Nature Research Reporting Summary linked to this article.

\section{Data availability}

Proteomics data were deposited in the ProteomeXchange database (PXD029002) through partner MassIVE (MSV000088204; https://massive.ucsd.edu/ProteoSAFe/dataset.jsp? task $=8 \mathrm{bc} 139 \mathrm{a} 2083 \mathrm{f} 481 \mathrm{fa} 7 \mathrm{bcb} 0 \mathrm{e} 62 \mathrm{c} 8 \mathrm{f} 1701)$. Other data generated or analyzed during this study are provided in the Supplementary Information and Source Data files. Source data are provided with this paper.

\section{Code availability}

All code and input data are currently stored in Github (https://github.com/csbio/ C.albicans-ml-pipeline) and Zenodo (https://doi.org/10.5281/zenodo.5565593).

Received: 5 August 2021; Accepted: 22 October 2021; Published online: 11 November 2021

\section{References}

1. Brown, G. D. et al. Hidden killers: human fungal infections. Sci. Transl. Med. 4, 165rv13 (2012).

2. Brown, G. D., Denning, D. W. \& Levitz, S. M. Tackling human fungal infections. Science 336, 647 (2012).

3. Fisher, M. C. et al. Threats posed by the fungal kingdom to humans, wildlife, and agriculture. mBio 11, e00449-20 (2020).

4. Pfaller, M. A. \& Diekema, D. J. Epidemiology of invasive candidiasis: a persistent public health problem. Clin. Microbiol. Rev. 20, 133-163 (2007).

5. Lockhart, S. R. Candida auris and multidrug resistance: defining the new normal. Fungal Genet. Biol. 131, 103243 (2019).

6. Lockhart, S. R. et al. Simultaneous emergence of multidrug-resistant Candida auris on 3 continents confirmed by whole-genome sequencing and epidemiological analyses. Clin. Infect. Dis. 64, 134-140 (2017).

7. Centers for Disease Control and Prevention. Antibiotic Resistance Threats in the United States (U.S. Department of Health and Human Services, 2019).

8. Lee, Y., Puumala, E., Robbins, N. \& Cowen, L. E. Antifungal drug resistance: molecular mechanisms in Candida albicans and beyond. Chem. Rev. 121, 3390-3411 (2020).

9. Perfect, J. R. The antifungal pipeline: a reality check. Nat. Rev. Drug Discov. 16, 603-616 (2017).

10. Haselbeck, R. et al. Comprehensive essential gene identification as a platform for novel anti-infective drug discovery. Curr. Pharm. Des. 8, 1155-1172 (2002).

11. Xue, A., Robbins, N. \& Cowen, L. E. Advances in fungal chemical genomics for the discovery of new antifungal agents. Ann. N. Y. Acad. Sci. 1496, 5-22 (2021).

12. Winzeler, E. A. et al. Functional characterization of the S. cerevisiae genome by gene deletion and parallel analysis. Science 285, 901-906 (1999).

13. Braun, B. R. et al. A human-curated annotation of the Candida albicans genome. PLoS Genet. 1, 36-57 (2005). 
14. Alby, K. \& Bennett, R. J. Sexual reproduction in the Candida clade: cryptic cycles, diverse mechanisms, and alternative functions. Cell Mol. Life Sci. 67, 3275-3285 (2010).

15. Bennett, R. J. \& Johnson, A. D. Mating in Candida albicans and the search for a sexual cycle. Annu. Rev. Microbiol. 59, 233-255 (2005).

16. Hickman, M. A. et al. The 'obligate diploid' Candida albicans forms matingcompetent haploids. Nature 494, 55-59 (2013).

17. Noble, S. M., French, S., Kohn, L. A., Chen, V. \& Johnson, A. D. Systematic screens of a Candida albicans homozygous deletion library decouple morphogenetic switching and pathogenicity. Nat. Genet. 42, 590-598 (2010).

18. Segal, E. S. et al. Gene essentiality analyzed by in vivo transposon mutagenesis and machine learning in a stable haploid isolate of Candida albicans. mBio 9, e02048-18 (2018).

19. Roemer, T. et al. Large-scale essential gene identification in Candida albicans and applications to antifungal drug discovery. Mol. Microbiol. 50, 167-181 (2003).

20. O’Meara, T. R. et al. Global analysis of fungal morphology exposes mechanisms of host cell escape. Nat. Commun. 6, 6741 (2015).

21. Skrzypek, M. S. et al. The Candida Genome Database (CGD): incorporation of Assembly 22, systematic identifiers and visualization of high throughput sequencing data. Nucleic Acids Res. 45, D592-D596 (2017).

22. O'Meara, T. R. \& O'Meara, M. J. DeORFanizing Candida albicans genes using coexpression. mSphere 6, e01245-20 (2021).

23. Wang, J. M., Bennett, R. J. \& Anderson, M. Z. The genome of the human pathogen Candida albicans is shaped by mutation and cryptic sexual recombination. mBio 9, e01205-e01218 (2018).

24. Costanzo, M. et al. A global genetic interaction network maps a wiring diagram of cellular function. Science 353, aaf1420 (2016).

25. Breiman, L. Random forests. Mach. Learn. 45, 5-32 (2001).

26. Roemer, T. \& Boone, C. Systems-level antimicrobial drug and drug synergy discovery. Nat. Chem. Biol. 9, 222-231 (2013).

27. O’Meara, T. R. et al. Global analysis of fungal morphology exposes mechanisms of host cell escape. Nat. Commun. 6, 6741 (2015).

28. Robbins, N., Wright, G. D. \& Cowen, L. E. Antifungal drugs: the current armamentarium and development of new agents. Microbiol. Spectr. 4, FUNK0002-FUNK-2016 (2016).

29. Wishart, D. S. et al. DrugBank 5.0: a major update to the DrugBank database for 2018. Nucleic Acids Res. 46, D1074-D1082 (2018).

30. Wang, Y. et al. Therapeutic target database 2020: enriched resource for facilitating research and early development of targeted therapeutics. Nucleic Acids Res. 48, D1031-D1041 (2020).

31. Kim, S. et al. PubChem in 2021: new data content and improved web interfaces. Nucleic Acids Res. 49, D1388-D1395 (2021).

32. Hornung, P. et al. Molecular architecture and connectivity of the budding yeast Mtw1 kinetochore complex. J. Mol. Biol. 405, 548-559 (2011).

33. Thakur, J. \& Sanyal, K. The essentiality of the fungus-specific Dam 1 complex is correlated with a one-kinetochore-one-microtubule interaction present throughout the cell cycle, independent of the nature of a centromere. Eukaryot. Cell 10, 1295-1305 (2011).

34. Li, J. M., Li, Y. \& Elledge, S. J. Genetic analysis of the kinetochore DASH complex reveals an antagonistic relationship with the ras/protein kinase A pathway and a novel subunit required for Ask1 association. Mol. Cell Biol. 25, 767-778 (2005).

35. Euskirchen, G. M. Nnflp, Dsn1p, Mtw1p, and Nsl1p: a new group of proteins important for chromosome segregation in Saccharomyces cerevisiae. Eukaryot. Cell 1, 229-240 (2002).

36. Cheeseman, I. M. et al. Implication of a novel multiprotein Dam1p complex in outer kinetochore function. J. Cell Biol. 155, 1137-1145 (2001).

37. Sanchez-Perez, I. et al. The DASH complex and Klp5/Klp6 kinesin coordinate bipolar chromosome attachment in fission yeast. EMBO J. 24, 2931-2943 (2005).

38. Burrack, L. S., Applen, S. E. \& Berman, J. The requirement for the Dam 1 complex is dependent upon the number of kinetochore proteins and microtubules. Curr. Biol. 21, 889-896 (2011).

39. Roy, B., Burrack, L. S., Lone, M. A., Berman, J. \& Sanyal, K. CaMtw1, a member of the evolutionarily conserved Mis12 kinetochore protein family, is required for efficient inner kinetochore assembly in the pathogenic yeast Candida albicans. Mol. Microbiol. 80, 14-32 (2011).

40. Berman, J. Morphogenesis and cell cycle progression in Candida albicans. Curr. Opin. Microbiol. 9, 595-601 (2006).

41. Noble, S. M., Gianetti, B. A. \& Witchley, J. N. Candida albicans cell-type switching and functional plasticity in the mammalian host. Nat. Rev. Microbiol. 15, 96-108 (2017).

42. Small, I., Peeters, N., Legeai, F. \& Lurin, C. Predotar: a tool for rapidly screening proteomes for N-terminal targeting sequences. Proteomics 4, 1581-1590 (2004).

43. Sun, N., Parrish, R. S., Calderone, R. A. \& Fonzi, W. A. Unique, diverged, and conserved mitochondrial functions influencing Candida albicans respiration. mBio 10, e00300-e00319 (2019).
44. Visacka, K. et al. Novel subfamily of mitochondrial HMG box-containing proteins: functional analysis of Gcf1p from Candida albicans. Microbiology 155, 1226-1240 (2009).

45. Diffley, J. F. \& Stillman, B. A close relative of the nuclear, chromosomal highmobility group protein HMG1 in yeast mitochondria. Proc. Natl Acad. Sci. USA 88, 7864-7868 (1991).

46. Larsson, N. G. et al. Mitochondrial transcription factor A is necessary for mtDNA maintenance and embryogenesis in mice. Nat. Genet. 18, 231-236 (1998).

47. Elantak, L. et al. The indispensable N-terminal half of eIF3j/HCR1 cooperates with its structurally conserved binding partner eIF3b/PRT1-RRM and with eIF1A in stringent AUG selection. J. Mol. Biol. 396, 1097-1116 (2010).

48. Ray, A., Bandyopadhyay, A., Matsumoto, T., Deng, H. \& Maitra, U. Fission yeast translation initiation factor 3 subunit eIF3h is not essential for global translation initiation, but deletion of eif $3 \mathrm{~h}+$ affects spore formation. Yeast $\mathbf{2 5}$, 809-823 (2008).

49. Roemer, T., Davies, J., Giaever, G. \& Nislow, C. Bugs, drugs and chemical genomics. Nat. Chem. Biol. 8, 46-56 (2012).

50. Beatty, K. E. et al. Fluorescence visualization of newly synthesized proteins in mammalian cells. Angew. Chem. Int. Ed. Engl. 45, 7364-7367 (2006).

51. Lee, A. Y. et al. Mapping the cellular response to small molecules using chemogenomic fitness signatures. Science 344, 208-211 (2014).

52. $\mathrm{Xu}, \mathrm{D}$. et al. Genome-wide fitness test and mechanism-of-action studies of inhibitory compounds in Candida albicans. PLoS Pathog. 3, e92 (2007).

53. Grant, T. D. et al. The structure of yeast glutaminyl-tRNA synthetase and modeling of its interaction with tRNA. J. Mol. Biol. 425, 2480-2493 (2013).

54. Sherlin, L. D. \& Perona, J. J. tRNA-dependent active site assembly in a class I aminoacyl-tRNA synthetase. Structure 11, 591-603 (2003).

55. Caplan, T. et al. Overcoming fungal echinocandin resistance through inhibition of the non-essential stress kinase Yck2. Cell Chem. Biol. 27, 269-282 (2020).

56. Liston, S. D., Whitesell, L., Kapoor, M., Shaw, K. J. \& Cowen, L. E. Enhanced efflux pump expression in Candida mutants results in decreased manogepix susceptibility. Antimicrob. Agents Chemother. 64, e00261-20 (2020).

57. Gale, A. N. et al. Identification of essential genes and fluconazole susceptibility genes in Candida glabrata by profiling hermes transposon insertions. G3 (Bethesda) 10, 3859-3870 (2020).

58. Arita, Y. et al. A genome-scale yeast library with inducible expression of individual genes. Mol. Syst. Biol. 17, e10207 (2021).

59. Becker, J. M. et al. Pathway analysis of Candida albicans survival and virulence determinants in a murine infection model. Proc. Natl Acad. Sci. USA 107, 22044-22049 (2010).

60. Witchley, J. N. et al. Candida albicans morphogenesis programs control the balance between gut commensalism and invasive infection. Cell Host Microbe 25, 432-443.e6 (2019).

61. Hillenmeyer, M. E. et al. The chemical genomic portrait of yeast: uncovering a phenotype for all genes. Science 320, 362-365 (2008).

62. Poulsen, B. E. et al. Defining the core essential genome of Pseudomonas aeruginosa. Proc. Natl Acad. Sci. USA 116, 10072-10080 (2019).

63. Galardini, M. et al. The impact of the genetic background on gene deletion phenotypes in Saccharomyces cerevisiae. Mol. Syst. Biol. 15, e8831 (2019).

64. Meraldi, P., McAinsh, A. D., Rheinbay, E. \& Sorger, P. K. Phylogenetic and structural analysis of centromeric DNA and kinetochore proteins. Genome Biol. 7, R23 (2006).

65. Jenni, S. \& Harrison, S. C. Structure of the DASH/Dam1 complex shows its role at the yeast kinetochore-microtubule interface. Science 360, 552-558 (2018).

66. Jeyaprakash, A. A. et al. Structural and functional organization of the Ska complex, a key component of the kinetochore-microtubule interface. $\mathrm{Mol}$. Cell 46, 274-286 (2012).

67. Eisen, M. B., Spellman, P. T., Brown, P. O. \& Botstein, D. Cluster analysis and display of genome-wide expression patterns. Proc. Natl Acad. Sci. USA 95, 14863-14868 (1998).

68. Ballouz, S., Verleyen, W. \& Gillis, J. Guidance for RNA-seq co-expression network construction and analysis: safety in numbers. Bioinformatics 31, 2123-2130 (2015)

69. Cairns, T. C. et al. Functional exploration of co-expression networks identifies a nexus for modulating protein and citric acid titres in Aspergillus niger submerged culture. Fungal Biol. Biotechnol. 6, 18 (2019).

70. Schape, P. et al. Updating genome annotation for the microbial cell factory Aspergillus niger using gene co-expression networks. Nucleic Acids Res. 47, 559-569 (2019).

71. Vincent, B. M. et al. A fungal-selective cytochrome bcl inhibitor impairs virulence and prevents the evolution of drug resistance. Cell Chem. Biol. 23 978-991 (2016).

72. She, X. et al. Fungal-specific subunits of the Candida albicans mitochondrial complex I drive diverse cell functions including cell wall synthesis. Cell Microbiol. 17, 1350-1364 (2015). 
73. Datt, M. \& Sharma, A. Novel and unique domains in aminoacyl-tRNA synthetases from human fungal pathogens Aspergillus niger, Candida albicans and Cryptococcus neoformans. BMC Genomics 5, 1069 (2014).

74. Francklyn, C. S. \& Mullen, P. Progress and challenges in aminoacyl-tRNA synthetase-based therapeutics. J. Biol. Chem. 294, 5365-5385 (2019).

75. Lv, P. C. \& Zhu, H. L. Aminoacyl-tRNA synthetase inhibitors as potent antibacterials. Curr. Med. Chem. 19, 3550-3563 (2012).

76. Nelson, J. et al. MOSAIC: a chemical-genetic interaction data repository and web resource for exploring chemical modes of action. Bioinformatics 34, 1251-1252 (2018).

77. Piotrowski, J. S. et al. Functional annotation of chemical libraries across diverse biological processes. Nat. Chem. Biol. 13, 982-993 (2017).

78. Pedregosa, F. Scikit-learn: machine learning in Python. J. Mach. Learn. Res 12, 2825-2830 (2011).

79. Min, K., Ichikawa, Y., Woolford, C. A. \& Mitchell, A. P. Candida albicans gene deletion with a transient CRISPR-Cas9 system. mSphere 1, e00130-16 (2016).

80. Lu, S. et al. CDD/SPARCLE: the conserved domain database in 2020. Nucleic Acids Res. 48, D265-D268 (2020).

81. Gish, W. \& States, D. J. Identification of protein coding regions by database similarity search. Nat. Genet. 3, 266-272 (1993).

82. Altschul, S. F., Gish, W., Miller, W., Myers, E. W. \& Lipman, D. J. Basic local alignment search tool. J. Mol. Biol. 215, 403-410 (1990).

83. Basenko, E. Y. et al. FungiDB: an integrated bioinformatic resource for fungi and oomycetes. J. Fungi 4, 39 (2018).

84. Chorostecki, U., Molina, M., Pryszcz, L. P. \& Gabaldon, T. MetaPhOrs 2.0: integrative, phylogeny-based inference of orthology and paralogy across the tree of life. Nucleic Acids Res. 48, W553-W557 (2020).

85. Sonnhammer, E. L. \& Ostlund, G. InParanoid 8: orthology analysis between 273 proteomes, mostly eukaryotic. Nucleic Acids Res. 43, D234-D239 (2015).

86. Cao, J. et al. The single-cell transcriptional landscape of mammalian organogenesis. Nature 566, 496-502 (2019).

87. McInnes, L., Healy, J. \& Melville, J. UMAP: Uniform manifold approximation and projection for dimension reduction. Preprint at https://arxiv.org/abs/ $1802.03426(2020)$

88. Traag, V. A., Waltman, L. \& van Eck, N. J. From Louvain to Leiden: guaranteeing well-connected communities. Sci. Rep. 9, 5233 (2019).

89. Wickham, H. ggplot2: Elegant Graphics for Data Analysis (Use R!) (Springer, 2016).

90. O’Meara, T. R. et al. Global proteomic analyses define an environmentally contingent Hsp90 interactome and reveal chaperone-dependent regulation of stress granule proteins and the R2TP complex in a fungal pathogen. PLoS Biol. 17, e3000358 (2019).

91. Xie, J. L. et al. Ydj1 governs fungal morphogenesis and stress response, and facilitates mitochondrial protein import via Mas1 and Mas2. Micro. Cell 4, 342-361 (2017).

92. Liu, G. et al. ProHits: integrated software for mass spectrometry-based interaction proteomics. Nat. Biotechnol. 28, 1015-1017 (2010).

93. Deutsch, E. W. et al. A guided tour of the Trans-Proteomic Pipeline. Proteomics 10, 1150-1159 (2010).

94. Shteynberg, D. et al. iProphet: multi-level integrative analysis of shotgun proteomic data improves peptide and protein identification rates and error estimates. Mol. Cell Proteom. 10, M111007690 (2011).

95. Teo, G. et al. SAINTexpress: improvements and additional features in Significance Analysis of INTeractome software. J. Proteom. 100, 37-43 (2014).

96. Xie, J. L., Singh-Babak, S. D. \& Cowen, L. E. Minimum Inhibitory Concentration (MIC) assay for antifungal drugs. Bio-Protocol 2, e252 (2012).

97. Kelley, L. A., Mezulis, S., Yates, C. M., Wass, M. N. \& Sternberg, M. J. The Phyre 2 web portal for protein modeling, prediction and analysis. Nat. Protoc. 10, 845-858 (2015).

98. Friesner, R. A. et al. Glide: a new approach for rapid, accurate docking and scoring. 1. Method and assessment of docking accuracy. J. Med. Chem. 47, 1739-1749 (2004).

99. Wapinski, I., Pfeffer, A., Friedman, N. \& Regev, A. Natural history and evolutionary principles of gene duplication in fungi. Nature 449, 54-61 (2007).

100. Harris, M. A., Lock, A., Bahler, J., Oliver, S. G. \& Wood, V. FYPO: the fission yeast phenotype ontology. Bioinformatics 29, 1671-1678 (2013).

101. Cherry, J. M. et al. Saccharomyces Genome Database: the genomics resource of budding yeast. Nucleic Acids Res. 40, D700-D705 (2012).

102. Dempster, J. M. et al. Agreement between two large pan-cancer CRISPR-Cas9 gene dependency data sets. Nat. Commun. 10, 5817 (2019).

103. Meyers, R. M. et al. Computational correction of copy number effect improves specificity of CRISPR-Cas9 essentiality screens in cancer cells. Nat. Genet. 49, 1779-1784 (2017).

\section{Acknowledgements}

We thank Merck and Genome Canada for making the original C. albicans DBC and GRACE mutant collections available. We thank all Cowen lab members for helpful discussions and all the current and past Cowen lab members for their assistance in expanding the GRACEv2 collection. We also thank Dr. Matthew Anderson for sharing the C. albicans SNP data, Dr. Sheena Li for the constant advice and support with chemical genomics, and Mami Yoshimura and Hiromi Kimura for technical support. Proteomics was performed at the Network Biology Collaborative Centre at the Lunenfeld-Tanenbaum Research Institute, a facility supported by Canada Foundation for Innovation funding, by the Ontarian Government, and by Genome Canada and Ontario Genomics (OGI-139). A.-C.G. is supported by the Canadian Institutes of Health Research (FDN 143301) and a Canada Research Chair, Tier 1, in Functional Proteomics. This research was partially supported by the Platform Project for Supporting Drug Discovery and Life Science Research from AMED under Grant Number JP20am0101086 (support number 0465). C.B. and Y.Y. are supported by a JSPS Grant-in-Aid for Scientific Research on Innovative Areas (17H06411). L.E.C. is supported by the Canadian Institutes of Health Research (CIHR) Foundation grant (FDN-154288) and a National Institutes of Health (NIH) R01 grant (R01AI127375); L.E.C. is a Canada Research Chair (Tier 1) in Microbial Genomics \& Infectious Disease and co-Director of the CIFAR Fungal Kingdom: Threats \& Opportunities program.

\section{Author contributions}

A.O.V., N.R., C.L.M. and L.E.C. conceptualized the study. A.-C.G., C.B., Y.Y. and L.E.C. obtained funding. B.V.S., J.H., Y.Y., A.-C.G., C.B., T.R.O., M.J.O., S.N., C.L.M. and L.E.C. provided resources for the study. C.F., X.Z., A.O.V., K.R.I., E.L., A.X., H.Y., N.M.R., C.W., Z.-Y.L., E.J.P. and S.L. carried out investigations. C.F., X.Z., A.O.V., K.R.I., E.L., A.X., S.L., T.R.O. and M.J.O. performed analysis and visualization. A.O.V., N.R., C.L.M. and L.E.C supervised the study. A.O.V., X.Z. and N.R. wrote the original draft and the manuscript, and all authors reviewed and edited the final manuscript.

\section{Competing interests}

L.E.C. is a co-founder and shareholder in Bright Angel Therapeutics, a platform company for the development of novel antifungal therapeutics. L.E.C. is a consultant for Boragen, a small-molecule development company focused on leveraging the unique chemical properties of boron chemistry for crop protection and animal health. All other authors declare no competing interests.

\section{Additional information}

Supplementary information The online version contains supplementary material available at https://doi.org/10.1038/s41467-021-26850-3.

Correspondence and requests for materials should be addressed to Chad L. Myers or Leah E. Cowen.

Peer review information Nature Communications thanks Ilse Jacobsen, David Perlin and the other, anonymous, reviewers for their contribution to the peer review of this work

Reprints and permission information is available at http://www.nature.com/reprints

Publisher's note Springer Nature remains neutral with regard to jurisdictional claims in published maps and institutional affiliations.

Open Access This article is licensed under a Creative Commons Attribution 4.0 International License, which permits use, sharing, adaptation, distribution and reproduction in any medium or format, as long as you give appropriate credit to the original author(s) and the source, provide a link to the Creative Commons license, and indicate if changes were made. The images or other third party material in this article are included in the article's Creative Commons license, unless indicated otherwise in a credit line to the material. If material is not included in the article's Creative Commons license and your intended use is not permitted by statutory regulation or exceeds the permitted use, you will need to obtain permission directly from the copyright holder. To view a copy of this license, visit http://creativecommons.org/ licenses/by/4.0/.

(c) The Author(s) 2021 